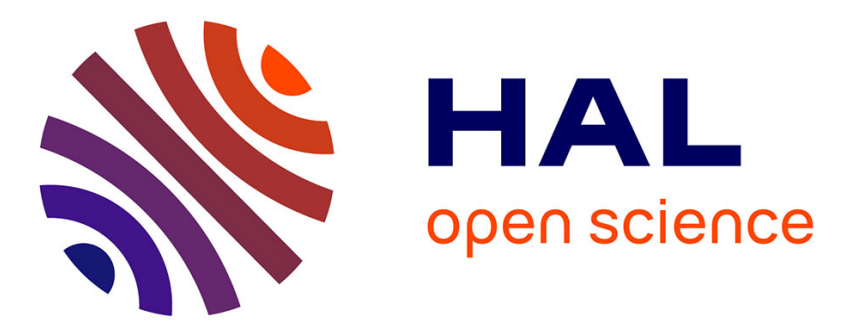

\title{
Reliability analysis of a satellite structure with a parametric and a non-parametric probabilistic model
}

M. Pellissitti, Evangéline Capiez-Lernout, H. Pradlwarter, Christian Soize, G.I. Schueller

\section{- To cite this version:}

M. Pellissitti, Evangéline Capiez-Lernout, H. Pradlwarter, Christian Soize, G.I. Schueller. Reliability analysis of a satellite structure with a parametric and a non-parametric probabilistic model. Computer Methods in Applied Mechanics and Engineering, 2008, 198 (2), pp.344-357. 10.1016/j.cma.2008.08.004 . hal-00686139

\section{HAL Id: hal-00686139 \\ https://hal.science/hal-00686139}

Submitted on 7 Apr 2012

HAL is a multi-disciplinary open access archive for the deposit and dissemination of scientific research documents, whether they are published or not. The documents may come from teaching and research institutions in France or abroad, or from public or private research centers.
L'archive ouverte pluridisciplinaire HAL, est destinée au dépôt et à la diffusion de documents scientifiques de niveau recherche, publiés ou non, émanant des établissements d'enseignement et de recherche français ou étrangers, des laboratoires publics ou privés. 


\section{Reliability analysis of a satellite structure with a parametric and a non-parametric probabilistic model}

Pellissetti, M. ${ }^{a}$, Capiez-Lernout, E. ${ }^{b}$, Pradlwarter, H. ${ }^{a}$, Soize,C. ${ }^{b}$, Schuëller, G.I..$^{a, \star}$

${ }^{a}$ University of Innsbruck, Institute of Engineering Mechanics, Technikerstrasse 13, 6020 Innsbruck, Austria

${ }^{b}$ Université Paris-Est, Modélisation et Simulation Multi Echelle (MSME FRE3160 CNRS), 5 Boulevard Descartes, 77455 Marne-la-Vallee, France

${ }^{\star}$ Corresponding author. Email: Mechanik@uibk.ac.at; Tel. +43 512507 6841; Fax: +43 5125072905 


\begin{abstract}
The reliability of a satellite structure subjected to harmonic base excitation in the low frequency range is analyzed with respect to the exceedance of critical frequency response thresholds. Both a parametric model of uncertainties and a more recently introduced nonparametric model are used to analyze the reliability, where the latter model in the present analysis captures the model uncertainties.

With both models, the probability of exceedance of given acceleration thresholds is estimated using Monte-Carlo simulation. To reduce the computational cost of the parametric model, a suitable meta-model is used instead.

The results indicate that for low levels of uncertainty in the damping, the non-parametric model provides significantly more pessimistic - and hence conservative - predictions about the exceedance probabilities. For high levels of damping uncertainty the opposite is the case.
\end{abstract}

Key words: Structural dynamics, frequency response functions, random uncertainties, parametric probabilistic model, non-parametric probabilistic model.

\title{
1 Introduction
}

\subsection{General remarks}

In structural dynamics, numerical models are used to perform dynamic analyses of complex mechanical systems. These models are usually based on the FE method and are constructed on the basis of the design of the structural system. Every manufacturing process is affected by some degree of variability and therefore the manufactured system is different from the designed system. These differences can have significant effects on the dynamics of the structure. Consequently, a deterministic model is usually not sufficient for a robust prediction of the dynamic response of the structure. In order to increase the robustness of the predictions, probabilistic models can be constructed on the basis of the deterministic model. Probabilistic models account for the un- 
certainties associated with the structure under consideration, by modelling some aspects of the structure as realizations of random variables or processes.

The random uncertainties can be split into two complementary classes. The uncertain parameters which are explicitly present both in the system design and in the mechanical-numerical model are referred to as system parameter uncertainties. On the other hand, due to simplifications in the construction of the mechanical-numerical model, there are uncertainties related to the designed system which are not explicitly present in the numerical model. This type of uncertainties is often referred to as model uncertainties.

In the parametric probabilistic approach the uncertain parameters of the mechanical-numerical model are treated as random quantities. The uncertain parameters include both geometrical parameters, such as components dimensions, and material properties, such as elasticity moduli. For each realization of the random parameters, the random finite element matrices result from a deterministic mapping of the random parameters. In the present paper the parametric model is used to capture the above mentioned system parameter uncertainties.

The non-parametric model of uncertainties has been introduced in [1,2]; in this approach, the generalised matrices resulting from the modal reduction of the deterministic FE model are replaced by random generalised matrices. The probabilistic description of these random matrices is constructed by using the maximum entropy principle under constraints defined by the available information and yields a new class of random matrices called the "positive definite ensemble" [3,4]. With such a formulation, the global dispersion level of each random matrix is controlled by a unique positive scalar which is called the dispersion parameter. In the present paper, the non-parametric model is used to account for model uncertainties.

The uncertainties associated with a numerical model are immediately related to the concept of risk, which combines the probability of an unfavourable event (e.g. failure of an engineered component or system) with the consequences of this event (e.g. human loss, injury, cost of repair, cost of claim management) $[5,6,7,8]$. For those that are responsible of the quality of engineered products, managing the risks associated with these products is of vital importance. Obviously, this importance then carries over to the availability of robust, quantitative estimates of 
the probability of the unfavourable events that may occur. (The evaluation of the consequences of such an event is outside the scope of our discussion.)

Given the high safety and quality standards required for the economic operation of engineering systems, in our case of structures, one usually attempts to keep the probability of an unfavourable event (failure) at very low levels. Consequently, failure is typically a rare event. This has implications for the mathematically formulated goal, which in this case is no longer the estimation of the probability distribution (or of its low-order moments) of some critical quantity of interest, but rather the statements derived from analysis involving the tails of the distribution.

With reference to the above stated distinction of uncertainties, it is obvious that the probability of an adverse event (and hence the associated risk) stems from both the uncertainty about the parameters of the system and the uncertainty about the assumptions in the model itself. This implies that the probability estimates are more complete - and hence more credible - if model uncertainties are accounted for.

In the context of structural dynamics, extensive work has been done in the past decades on the uncertainty analysis of engineering structures, in which the uncertainty was assumed to relate exclusively to the parameters of the model. In the literature, numerous works both on uncertainty quantification, which are aiming at the characterization of the (approximate) probability distribution and the low-order statistics (see e.g. $[9,10,11,12,13,14,15,16,17])$, and on the estimation of (small) failure probabilities (see e.g. $[18,19,20,21,22]$ ) may be found.

On the other hand, a much smaller number of publications deal with model uncertainties in the structural analysis. Besides the here adopted non-parametric approach, past attempts include the treatment of model uncertainties in a Bayesian framework (see e.g. $[23,24,25]$ ) and the specific analysis of the impact of model uncertainties on the failure probability (see e.g. $[26,27,28]$ ). It should be noted that for the consideration of model uncertainties with Bayesian methods, it is required that experimental results be available. This requirement is frequently not fulfilled in the context of real-life engineering analysis and design tasks. In such situations, the non-parametric model still remains applicable, as it does not necessarily require the availability of experimental results. 
The novel contribution of this paper is a study on the effect of including model uncertainties in the analysis of the failure probability of complex structures under dynamic excitation. The model uncertainties are represented here with a non-parametric model that is constructed on the basis of the FE model of a satellite with approximately 120,000 DOF's [29]. More specifically, the non-parametric model is calibrated with respect to a parametric model of uncertainties, i.e. a probabilistic model in which the parameters of the nominal finite element model of the structure are modelled as random variables.

Given that the results presented in this paper indicate a very different sensitivity of the predicted failure probability to the damping uncertainty, particular emphasis is given to this aspect. A fullscale parametric study has been performed, in order to study the failure probability estimates for various levels of uncertainty in the damping.

\subsection{Notation}

The following notation is adopted in the present paper:

- A real or complex deterministic scalar is denoted by a lower case letter (for instance $f$ ).

- A real or complex-valued random variable is denoted by an upper case letter (for instance $F$ ).

- A real or complex deterministic vector is denoted by a boldface lower case letter (for instance $\left.\mathbf{f}=\left[f_{1} f_{2} \ldots f_{n}\right]^{T}\right)$

- A real or complex-valued random vector is denoted by a boldface upper case letter (for instance $\mathbf{F}=\left[\begin{array}{llll}F_{1} & F_{2} & \ldots & F_{n}\end{array}\right]^{T}$ )

- A real or complex deterministic matrix is denoted by an upper case letter between brackets (for instance $[A]$ )

- A real or complex-valued random matrix is denoted by a boldface upper case letter between brackets (for instance $[\mathbf{A}]$ )

- All the deterministic quantities related to the mean FE model are underlined (for instance $\underline{f}, \underline{\mathbf{f}},[\underline{A}])$. More specifically, the term "mean model" denotes the deterministic model obtained when all its physical parameters are fixed at their mean value and is hence equivalent to the term "nominal model". 
- Frequencies are denoted as $\bar{\omega}$ in the case of excitation frequencies, and as $\omega$ (or with an index, e.g. $\left.\omega_{j}\right)$ in the case of eigenfrequencies.

\section{Method of analysis}

\subsection{Frequency response analysis}

In the present analysis the linear vibrations of a free structure are considered, around a static equilibrium configuration considered as a natural state without prestresses in the low frequency band $\mathbb{B}$. For all $\bar{\omega} \in \mathbb{B}$, the mean finite element matrix equation of the structure is,

$$
\left(-\bar{\omega}^{2}[\underline{M}]+i \bar{\omega}[\underline{D}]+[\underline{K}]\right) \underline{\mathbf{u}}(\bar{\omega})=\underline{\mathbf{f}}(\bar{\omega})
$$

in which $\underline{\mathbf{u}}(\bar{\omega})$ and $\underline{\mathbf{f}}(\bar{\omega})$ are the $\mathbb{C}^{m}$ vectors of the DOF's and of the external forces, respectively. Since the structure has a free boundary, the mean mass matrix $[\underline{M}]$ is a positive-definite symmetric $(m \times m)$ real matrix and the mean damping and stiffness matrices are positive semidefinite symmetric $(m \times m)$ real matrices. Furthermore, it is assumed that the kernel of mean matrices $[\underline{D}]$ and $[\underline{K}]$ is identical, constituted of $r$ rigid-body modes with $0 \leq r \leq 6$ denoted as $\underline{\varphi}_{1}, \ldots, \underline{\varphi}_{r}$.

\subsection{Parametric probabilistic model}

Let $\underline{\mathbf{x}}=\left(\underline{x}_{1}, \ldots, \underline{x}_{d}\right)$ be an $\mathbb{R}^{d}$ vector whose components correspond to parameters of the structure, such as geometrical parameters of the structure, coefficients of the elasticity tensor, mass densities. Clearly, the entries of the mass, damping and stiffness matrices of the FE model are functions of these parameters.

Assuming that the parameters collected in $\underline{\mathbf{x}}$ are affected by uncertainty, we then introduce the $\mathbb{R}^{d}$-valued random variable $\mathbf{X}=\left(X_{1}, \ldots, X_{d}\right)$. The system of equations associated with this 
random finite element model has then the form,

$$
\left(-\bar{\omega}^{2}\left[\mathbf{M}^{\mathrm{par}}\right]+i \bar{\omega}\left[\mathbf{D}^{\mathrm{par}}\right]+\left[\mathbf{K}^{\mathrm{par}}\right]\right) \mathbf{U}^{\mathrm{par}}(\bar{\omega})=\underline{\mathbf{f}}(\bar{\omega})
$$

in which $\mathbf{U}^{\text {par }}(\bar{\omega})$ is the $\mathbb{C}^{m}$-valued random vector of the DOFs and where $\left[\mathbf{M}^{\text {par }}\right]=[M(\mathbf{X})]$, and $\left[\mathbf{D}^{\mathrm{par}}\right]=[D(\mathbf{X})],\left[\mathbf{K}^{\mathrm{par}}\right]=[K(\mathbf{X})]$ are the random finite element mass, damping and stiffness matrices with values in the set of the positive-definite symmetric $(m \times m)$ real matrices and in the set of the positive semidefinite symmetric $(m \times m)$ real matrices. The components $\left\{X_{i}, i \in\{1, \ldots, d\}\right\}$ of the random vector $\mathbf{X}$ are random variables with a given joint cumulative distribution function $(\mathrm{CDF})$,

$$
F_{\mathbf{X}}(\mathbf{x})=P\left[X_{1} \leq x_{1}, X_{2} \leq x_{2}, \ldots, X_{d} \leq x_{d}\right]
$$

and with mean values $\mu_{X_{i}}=\underline{x}_{i}$ and standard deviations $\sigma_{X_{i}}$.

For large systems the solution of Eq. 2 can be computed efficiently with a reduced version of the equation,

$$
\left(-\bar{\omega}^{2}\left[\mathbf{M}_{\mathrm{red}}^{\mathrm{par}}\right]+i \bar{\omega}\left[\mathbf{D}_{\mathrm{red}}^{\mathrm{par}}\right]+\left[\mathbf{K}_{\mathrm{red}}^{\mathrm{par}}\right]\right) \mathbf{Q}^{\mathrm{par}}(\bar{\omega})=\mathcal{F}(\bar{\omega}),
$$

in which $\left[\mathbf{M}_{\text {red }}^{\text {par }}\right]$, $\left[\mathbf{D}_{\text {red }}^{\text {par }}\right]$ and $\left[\mathbf{K}_{\text {red }}^{\text {par }}\right]$ are the diagonal random reduced mass, damping and stiffness matrices $(n \times n)$,

$$
\left[\mathbf{M}_{\mathrm{red}}^{\mathrm{par}}\right]=\left[\boldsymbol{\Phi}^{T}\right]\left[\mathbf{M}^{\mathrm{par}}\right][\boldsymbol{\Phi}], \quad\left[\mathbf{D}_{\mathrm{red}}^{\mathrm{par}}\right]=\left[\boldsymbol{\Phi}^{T}\right]\left[\mathbf{D}^{\mathrm{par}}\right][\boldsymbol{\Phi}], \quad\left[\mathbf{K}_{\mathrm{red}}^{\mathrm{par}}\right]=\left[\boldsymbol{\Phi}^{T}\right]\left[\mathbf{K}^{\mathrm{par}}\right][\boldsymbol{\Phi}] .
$$

In the above equation, $[\boldsymbol{\Phi}]$ is an $(m \times n)$ real matrix whose columns are the $n \lll m$ eigenvectors $\boldsymbol{\Phi}_{\alpha}$ related to the $n$ strictly positive lowest eigenfrequencies $\Lambda_{\alpha}=\Omega_{\alpha}^{2}$, corresponding to the generalized eigenvalue problem,

$$
\left[\mathbf{K}^{\mathrm{par}}\right] \Phi=\Lambda\left[\mathbf{M}^{\mathrm{par}}\right] \Phi
$$

For mass-normalized eigenvectors and assuming proportional damping, the diagonal matrices in Eq. (5) are specified as follows,

$$
\left[\mathbf{M}_{\mathrm{red}}^{\mathrm{par}}\right]=\left[I_{n}\right], \quad\left[\mathbf{D}_{\mathrm{red}}^{\mathrm{par}}\right]_{i j}=2 \Xi_{j} \Omega_{j} \delta_{i j}, \quad\left[\mathbf{K}_{\mathrm{red}}^{\mathrm{par}}\right]_{i j}=\Omega_{j}^{2} \delta_{i j}
$$


where $\Xi_{j}$ is the $j$-th modal damping ratio and $\delta_{i j}$ is the Kronecker delta. It should be noted that in the present paper the modal damping ratios are considered to be random variables $\Xi_{j}=\Xi_{j}(\mathbf{X})$ and are thus capitalized according to the notation described in section 1.2.

Considering that the generalized force $\mathcal{F}(\bar{\omega})=[\Phi]^{T} \underline{\mathbf{f}}(\bar{\omega})$, the $\mathbb{C}^{n}$-valued random vector of the generalised coordinates $\mathbf{Q}^{\text {par }}(\bar{\omega})$ can be computed from Eq. (4),

$$
Q_{j}^{\mathrm{par}}(\bar{\omega})=\frac{\mathcal{F}_{j}(\bar{\omega})}{-\bar{\omega}^{2}+i 2 \bar{\omega} \Omega_{j} \Xi_{j}+\Omega_{j}^{2}}, \quad j=1, \ldots, n .
$$

The solution vector $\mathbf{U}^{\mathrm{par}}(\bar{\omega})$ can then be reconstructed as follows,

$$
\mathbf{U}^{\mathrm{par}}(\bar{\omega})=[\boldsymbol{\Phi}] \mathbf{Q}^{\mathrm{par}}(\bar{\omega}), \quad \text { i.e. } \quad U_{i}^{\mathrm{par}}(\bar{\omega})=\sum_{j=1}^{n} \frac{[\boldsymbol{\Phi}]_{i j} \mathcal{F}_{j}(\bar{\omega})}{-\bar{\omega}^{2}+i 2 \bar{\omega} \Omega_{j} \Xi_{j}+\Omega_{j}^{2}} .
$$

Meta-model of the parametric probabilistic model Given that the reliability analysis pursued in this paper requires a very large number of samples of the response quantities of interest, it would be prohibitive to use the full finite element model of the structure to produce the samples of the parametric model of uncertainties. Consequently, a meta-model is constructed, which is supposed to mimic the behaviour of the full model for a given sample $\mathbf{X}^{(k)}$ of the uncertain input parameters, at a greatly reduced computational cost. Denoting by $t_{\text {Meta }}$ and $t_{\text {Full }}$ the CPUtimes required to compute one sample of the response using the meta-model and the full FE model, respectively, the meta-model should have the following properties,

$$
R_{\text {Meta }}^{(k)}=R_{\text {Meta }}\left(\mathbf{X}^{(k)}\right) \approx R_{\text {Full }}^{(k)}=R_{\text {Full }}\left(\mathbf{X}^{(k)}\right) \quad \forall k \in(1,2, \ldots N), \quad t_{\text {Meta }} \ll t_{\text {Full }}
$$

where $R_{\text {Meta }}$ and $R_{\text {Full }}$ are the value of a generic response quantity of interest predicted by the meta-model and the full model, respectively. With reference to Eq. (9), in the parametric probabilistic model such a quantity derived from the frequency response $\mathbf{U}^{\mathrm{par}}(\bar{\omega})$ can be computed with the following expression,

$$
R_{\text {Full }}(\bar{\omega})=\sum_{j=1}^{n} \frac{C_{j} \bar{\omega}^{p}}{-\bar{\omega}^{2}+i 2 \bar{\omega} \Omega_{j} \Xi_{j}+\Omega_{j}^{2}}, \quad p \in\{0,1,2\},
$$


where the random scalar $C_{j}=C_{j}\left(\boldsymbol{\Phi}_{j}, \mathcal{F}_{j},\left[\mathbf{M}^{\mathrm{par}}\right],\left[\mathbf{D}^{\mathrm{par}}\right],\left[\mathbf{K}^{\mathrm{par}}\right]\right)$ depends on the corresponding eigenvector $\boldsymbol{\Phi}_{j}$, on the generalized force $\mathcal{F}_{j}$ and possibly also on the matrices $\left[\mathbf{M}^{\mathrm{par}}\right],\left[\mathbf{D}^{\mathrm{par}}\right],\left[\mathbf{K}^{\mathrm{par}}\right]$. The value of the exponent $p$ depends on the response quantity, e.g. for accelerations $p=2$.

Since the adopted meta-model has mainly the role of speeding up the computations performed with the parametric probabilistic model, its presentation is attached in appendix A.

\section{$2.3 \quad$ Non-parametric model of uncertainties}

It is recalled that the main idea of the non-parametric probabilistic model of random uncertainties consists in replacing the generalised matrices of a mean reduced matrix model of the structure by random matrices whose probability model is constructed with the maximum entropy principle. In particular, the theoretical construction and the physical concepts of this recent probabilistic approach are detailed in $[1,2,4,3]$. Below, the main steps for establishing the random matrix equations are summarised.

\subsubsection{Mean reduced matrix model}

Given the focus on the elastic motion of the structure, the $(m \times n)$ real matrix $[\underline{\Phi}]$ is introduced, whose columns are the $n \lll m$ eigenvectors $\underline{\varphi}_{\alpha}$ related to the $n$ strictly positive lowest eigenfrequencies $\underline{\lambda}_{\alpha}=\underline{\omega}_{\alpha}^{2}$. The mean reduced matrix model can then be written as,

$$
\underline{\mathbf{u}}(\bar{\omega})=[\underline{\Phi}] \underline{\mathbf{q}}(\bar{\omega})
$$

in which $\underline{\mathbf{q}}(\bar{\omega})$ is the $\mathbb{C}^{n}$ vector of the generalised coordinates solution of the mean reduced equation

$$
\left(-\bar{\omega}^{2}\left[\underline{M}_{\mathrm{red}}\right]+i \bar{\omega}\left[\underline{D}_{\mathrm{red}}\right]+\left[\underline{K}_{\mathrm{red}}\right]\right) \underline{\mathbf{q}}(\bar{\omega})=\underline{\mathcal{F}}(\bar{\omega}) .
$$

In the above Eq. $\underline{\mathcal{F}}(\bar{\omega})=[\underline{\Phi}]^{T} \underline{\mathbf{f}}(\bar{\omega})$ is the $\mathbb{C}^{n}$ vector of the generalised forces and the mean reduced mass, damping and stiffness matrices $\left[\underline{M}_{\mathrm{red}}\right]=[\underline{\Phi}]^{T}[\underline{M}][\underline{\Phi}],\left[\underline{D}_{\mathrm{red}}\right]=[\underline{\Phi}]^{T}[\underline{D}][\underline{\Phi}]$ and $\left[\underline{K}_{\mathrm{red}}\right]=[\underline{\Phi}]^{T}[\underline{K}][\underline{\Phi}]$ are positive-definite symmetric $(n \times n)$ real matrices. 


\subsubsection{Construction of the non-parametric model of random uncertainties}

The non-parametric model of random uncertainties yields the random matrix equation

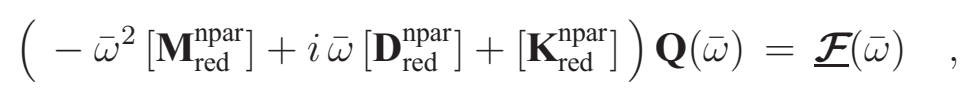

in which $\left[\mathbf{M}_{\text {red }}^{\text {npar }}\right]$, $\left[\mathbf{D}_{\text {red }}^{\text {npar }}\right]$ and $\left[\mathbf{K}_{\text {red }}^{\text {npar }}\right]$ are positive-definite symmetric $(n \times n)$ real-valued matrices corresponding to the random reduced mass, damping and stiffness matrices and where $\mathbf{Q}(\bar{\omega})$ is the $\mathbb{C}^{n}$-valued random vector of the random generalised coordinates. The $\mathbb{C}^{m}$-valued random vector $\mathbf{U}^{\text {npar }}(\bar{\omega})$ is thus reconstructed by

$$
\mathbf{U}^{\mathrm{npar}}(\bar{\omega})=\left[\underline{\Phi}^{n}\right] \mathbf{Q}(\bar{\omega})
$$

\subsubsection{Probability model of the random matrices}

The non-parametric probabilistic approach requires the normalisation of the mean reduced matrices such that $\left[\underline{M}_{\mathrm{red}}\right]=\left[\underline{L}_{M}\right]^{T}\left[\underline{L}_{M}\right],\left[\underline{D}_{\mathrm{red}}\right]=\left[\underline{L}_{D}\right]^{T}\left[\underline{L}_{D}\right]$ and $\left[\underline{K}_{\mathrm{red}}\right]=\left[\underline{L}_{K}\right]^{T}\left[\underline{L}_{K}\right]$, in which $\left[\underline{L}_{M}\right],\left[\underline{L}_{D}\right]$ and $\left[\underline{L}_{K}\right]$ are diagonal $(n \times n)$ real matrices. Each random matrix is written as

$$
\begin{aligned}
{\left[\mathbf{M}_{\text {red }}^{\text {npar }}\right] } & =\left[\underline{L}_{M}\right]^{T}\left[\mathbf{G}_{M}\right]\left[\underline{L}_{M}\right] \\
{\left[\mathbf{D}_{\text {rad }}^{\text {nar }}\right] } & =\left[\underline{L}_{D}\right]^{T}\left[\mathbf{G}_{D}\right]\left[\underline{L}_{D}\right] \\
{\left[\mathbf{K}_{\text {red }}^{\text {npar }}\right] } & =\left[\underline{L}_{K}\right]^{T}\left[\mathbf{G}_{K}\right]\left[\underline{L}_{K}\right] .
\end{aligned}
$$

The probability distribution of the random matrices $\left[\mathbf{G}_{M}\right],\left[\mathbf{G}_{D}\right]$ and $\left[\mathbf{G}_{K}\right]$ is derived from the maximum entropy principle issued from the information theory [30] with the available information [1]. It can be shown that random matrices $\left[\mathbf{G}_{M}\right],\left[\mathbf{G}_{D}\right]$ and $\left[\mathbf{G}_{K}\right]$ are independent random variables whose dispersion level can be controlled by the positive real parameters $\delta_{M}, \delta_{D}$ and $\delta_{K}$ which are independent of the dimension $n$. The probability distribution of these random matrices and the generator of independent realizations are summarized in the appendix B. 
2.4 Identification of the dispersion parameters related to the non-parametric approach with respect to the parametric approach

In this section, the identification strategy of the dispersion parameters $\delta_{M}, \delta_{D}$ and $\delta_{K}$, which control the dispersion of each random matrix issued from the non-parametric probabilistic approach, is described. Essentially, this strategy aims at introducing the same level of uncertainty in some aspects of the numerical model which can be expected to be affected by a small degree of model uncertainty. This is for instance usually the case for the first eigenfrequency of a numerical model, after applying a suitable updating procedure [31].

In the choice of a suitable parameter to be used in the definition of the cost function utilized for the calibration, it should be noted that numerical models for structural dynamics are typically validated with special attention that the model captures the behavior of the physical reality very well for the first eigenmode. Consequently, the model uncertainties may be deemed as having a minimum impact on the first eigenmode, compared to other results of the modal analysis.

The consideration of the unavoidable uncertainties in the physical parameters of the numerical model will lead to a commensurate scatter in the modal parameters. Again, in view of the above argument, the scatter of the first eigenfrequency can in most cases be expected to be the one measure of the variability, which best represents the scatter observed in an ensemble of realizations of the physical structure (which in most cases are not available, especially for one-of-a-kind structures such as the satellite considered in this study). Given that - in most practical cases of interest - the confidence in the characterization of the scatter in the first eigenfrequency is hence superior to that of other modal parameters, it is reasonable to calibrate the non-parametric model such that it produces the same scatter in the first eigenfrequency [29].

Let $\Lambda_{\alpha}^{\mathrm{par}}$ and $\Lambda_{\alpha}^{\text {npar }}$ be the first non zero random eigenvalues obtained with the parametric and the non-parametric model of random uncertainties, respectively. The probability density functions (PDFs) of random eigenvalues $\Lambda_{1}^{\mathrm{par}}$ and $\Lambda_{1}^{\mathrm{npar}}$, denoted as $p_{1}^{\text {par }}(\lambda)$ and $p_{\Lambda_{1}^{\mathrm{npar}}}(\lambda)$, are compared in the least square sense. The two-dimensional cost function $J\left(\delta_{M}, \delta_{K}\right)$ is then introduced such 
that,

$$
J\left(\delta_{M}, \delta_{K}\right)=\frac{\left\|p_{\Lambda_{1}^{\mathrm{npar}}}\left(\delta_{M}, \delta_{K}\right)-p_{\Lambda_{1}^{\mathrm{par}}}\right\|_{\mathcal{L}^{2}}}{\left\|p_{\Lambda_{1}^{\mathrm{par}}}\right\|_{\mathcal{L}^{2}}},
$$

in which the norm $\|f\|_{\mathcal{L}^{2}}$ is given by

$$
\|f\|_{\mathcal{L}^{2}}=\left(\int_{\mathbb{R}}|f(\mathbf{x})|^{2} d \mathbf{x}\right)^{1 / 2} .
$$

The identification is carried out such that parameters $\delta_{M}$ and $\delta_{K}$ minimise the cost function and are hence the solution of the optimization problem,

$$
\min _{\delta_{M}, \delta_{K}} J\left(\delta_{M}, \delta_{K}\right)
$$

The dispersion parameter $\delta_{D}$ is identified separately by using the identification method proposed in [32]. Let $\left[\mathbf{D}_{\mathrm{red}}^{\mathrm{par}}\right]$ be the random dissipation matrix issued from the random reduced model related to the parametric probabilistic model. The dispersion parameter $\delta_{D}$ is computed as follows,

$$
\delta_{D}=\sqrt{\frac{W_{D}^{\mathrm{par}}(n+1)}{\operatorname{tr}\left(\left[\underline{D}_{\mathrm{red}}\right]\right)^{2}+\operatorname{tr}\left(\left[\underline{D}_{\mathrm{red}}\right]^{2}\right)}},
$$

in which $W_{D}^{\mathrm{par}}$ is given by

$$
W_{D}^{\mathrm{par}}=E\left\{\left\|\left[\mathbf{D}_{\mathrm{red}}^{\mathrm{par}}\right]-\left[\underline{D}_{\mathrm{red}}\right]\right\|_{F}^{2}\right\}
$$

and where $\|[A]\|_{F}^{2}=\operatorname{tr}\left([A][A]^{T}\right)$.

\subsection{Reliability analysis}

\subsubsection{General remarks}

The assessment of the reliability of structures requires a quantitative definition of failure. For this purpose a so-called performance function $g(\mathbf{X})$ is defined, which characterizes the state of the structure and which is therefore a function of the vector of the uncertain parameters $\mathrm{X}$,

$$
g(\mathbf{X}) \text { such that }\left\{\begin{array}{l}
g(\mathbf{X})>0 \Longleftrightarrow \mathbf{X} \in S \\
g(\mathbf{X}) \leq 0 \Longleftrightarrow \mathbf{X} \in F \\
g(\mathbf{X})=0 \Longleftrightarrow \text { limit state }
\end{array}\right.
$$


where $S$ and $F$ denote the safe set and the failure set, respectively. The reliability of a structure can then be quantified by its complementary quantity, the probability of failure $p_{F}$,

$$
p_{F}=P[\mathbf{X} \in F]=\int \mathbb{1}_{F}(\mathbf{x}) f_{\mathbf{X}}(\mathbf{x}) d \mathbf{x}, \quad \mathbb{1}_{F}(\mathbf{X})=\left\{\begin{array}{l}
0 \Longleftrightarrow \mathbf{X} \in S \\
1 \Longleftrightarrow \mathbf{X} \in F,
\end{array}\right.
$$

Computing this integral is a complex task for complex structural systems, because the evaluation of the indicator function is usually time consuming, since it is usually necessary to perform a full FE analysis in order to determine whether a realization of the input parameters leads to failure. In the present work Monte-Carlo simulation $[33,20]$ has been adopted, which is the most robust and generally applicable method for estimating $p_{F}$.

\subsubsection{Monte Carlo Simulation}

The Monte Carlo estimator $\hat{p}_{F}$ for the probability of failure $p_{F}=P[F]$, where $F$ denotes the failure event, has the form,

$$
\hat{p}_{F}=\frac{1}{N} \sum_{k=1}^{N} \mathbb{1}_{F}\left(\mathbf{X}^{(k)}\right) .
$$

In the above equation, $N$ is the number of samples and $\mathrm{X}^{(k)}$ denotes the $k$-th realization of the set of input variables. The variance and the coefficient of variation (C.o.V.) of this estimator are as follows,

$$
\operatorname{Var}\left[\hat{p}_{F}\right]=\frac{1-p_{F}}{p_{F} N}, \quad \operatorname{CoV}{\hat{\hat{p}_{F}}}_{F}=\sqrt{\operatorname{Var}\left[\hat{p}_{F}\right]} / p_{F}=\sqrt{\left(1-p_{F}\right) / N p_{F}}
$$

and represent a measure of the accuracy of the estimate. It should be noted that $C o V_{\hat{p}_{F}}$ is independent of the dimensionality of the random vector $\mathrm{X}$. Besides the estimate of $\mathrm{CoV}_{\hat{p}_{F}}$, the accuracy of the Monte Carlo estimator $\hat{p}_{F}$ can be quantified by constructing the associated confidence interval based on Chebyshev's inequality,

$$
P\left[\hat{p}_{F}-\epsilon<p_{F}<\hat{p}_{F}+\epsilon\right] \geq \alpha, \quad \text { where } \quad \alpha=1-\frac{\sigma_{\hat{p}_{F}}^{2}}{\epsilon^{2}} .
$$


From the above, the limits of the confidence interval can be estimated in terms of $\epsilon$ for any specified confidence level $\alpha$ (e.g. $\alpha=90 \%$ ),

$$
\epsilon \approx \sqrt{\frac{\hat{p}_{F}\left(1-\hat{p}_{F}\right)}{N(1-\alpha)}} .
$$

\subsubsection{Computational efficiency}

Eq. (27) shows that for the accurate estimation of small $p_{F}$ 's a high number (proportional to $\left.1 / p_{F}\right)$ of samples is needed. In this work the computational efficiency has been improved by using i.) a meta model (section A), and ii.) parallel processing (section 3.8.2). An additional measure for reducing the computational costs would be afforded by advanced MCS techniques $[19,20]$, which aim at reducing the variance of the estimator of $p_{F}$.

\section{Reliability analysis of a satellite structure subjected to base excitation}

\subsection{General remarks}

The purpose of the following analysis is to study the reliability of a satellite structure subjected to a base excitation, under consideration of the uncertainties associated with the numerical model of the structure. The investigated structure corresponds to the INTEGRAL satellite of the European Space Agency (ESA), used for astrophysical research missions (INTErnational Gamma Ray Astrophysics Laboratory) [34]. In the present study the structural reliability has been defined with respect to the exceedance of given acceleration thresholds of the FRF in the low frequency range.

Both a parametric (section 3.3) and a non-parametric model (section 3.4) have been used to assess the reliability. The obtained results show that the reliability computed with the two models differs greatly (section 3.6). As the magnitude of this difference varies depending on the magnitude of the uncertainty in the damping, a parameter study has been performed (section 3.7), 
in order to investigate this dependence in more detail.

\subsection{Finite element model description}

The finite element model underlying this analysis [35,36,37] is encoded for the commercial FE code MSC.Nastran [38] and is shown in Fig. 1. The model has approximately 120,000 DOFs; triangular and quadrilateral shell elements have been used to model the numerous panel-like components. Composite material properties have been introduced wherever applicable. The instruments have been included in the model in reduced form, using the Craig-Bampton method as well as the Guyan-reduction technique. Linear elastic behavior is assumed throughout the model. A harmonic base excitation with the frequency $\bar{f} \in[5 \mathrm{~Hz}, 25 \mathrm{~Hz}]$ is imposed at the sup-

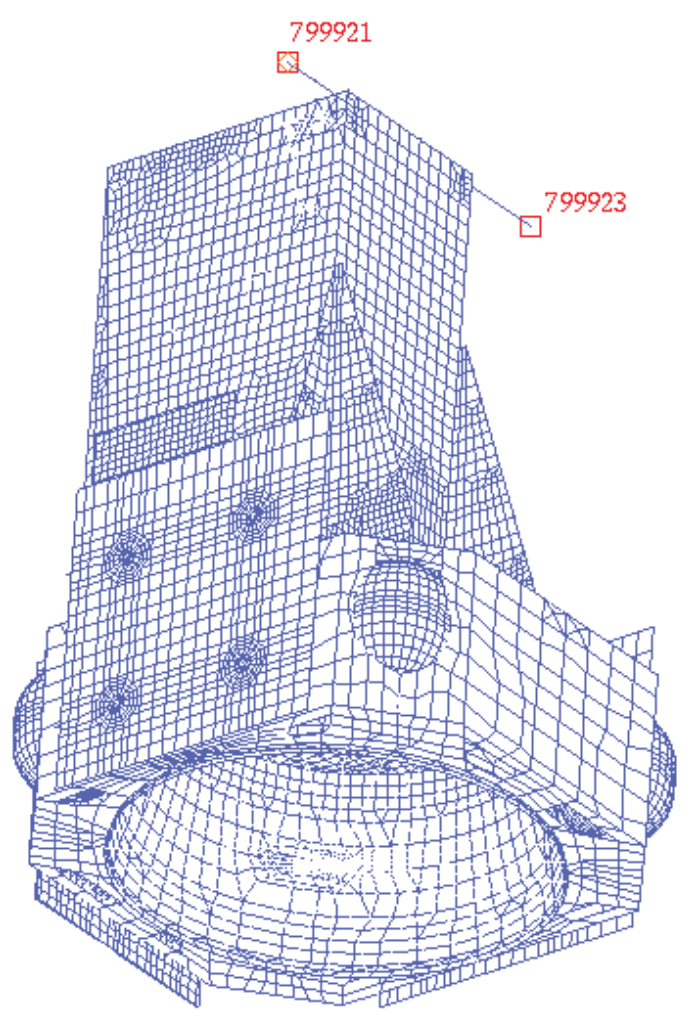

Fig. 1. Finite element model of the INTEGRAL satellite (courtesy of ESA/ESTEC)

port, which consists in the circular ring at the bottom of the satellite structure, as shown in Fig. 1. The magnitude of the imposed acceleration is $1 g$; the direction of the excitation is horizontal 
and parallel to the axis of symmetry of the satellite.

Energy dissipation is modelled at system level, by assuming proportional damping. More specifically the damping ratio is assumed as $\zeta=1.5 \%$ (of critical damping) for all modes with a natural frequency below $30 \mathrm{~Hz}$ (i.e. for the first four modes) and as $\zeta=2.5 \%$ for the modes with a natural frequency above $30 \mathrm{~Hz}$.

\subsection{Parametric probabilistic model}

As mentioned in section 2.2, in the parametric probabilistic model the uncertain parameters of the nominal FE model are treated as random variables, with a given distribution (see Eq. (3)). In this study both material and geometric properties of the FE model have been assumed to be uncertain; this includes beam section dimensions, composite material fibre orientation, non structural masses, elastic moduli etc.

For structures as complex as the here investigated satellite it is very difficult to make an a-priori assessment of whether the uncertainty in a given parameter is influential and should be considered or whether it can be neglected. Therefore the parameters have been classified into various types and for each occurrence of a certain parameter type, an independent random variable has been defined.

The assumed distribution type and the magnitude of the variability depend on the parameter type and are reported in Table 1. For most uncertain parameters a normal distribution has been assumed, which has been truncated to avoid negative values of the parameters, which are physically strictly positive. Since in the case of damping the associated uncertainty is significantly larger (up to $\mathbf{5 0 \%}$ in the present study), a log-normal distribution has been assumed. All uncertain parameters have been assumed to be mutually independent. The probabilistic modeling approach summarized in Table 1 leads to a total of 1319 independent random variables with coefficients of variation between $4 \%$ and $12 \%$. It should be noted that the magnitude of the scatter has been selected on the basis of data available in the literature $[39,40,41,42]$. 
Table 1

Assumptions for the probability distribution / C.o.V. of the uncertain parameters in the parametric model

\begin{tabular}{|l|l|l|l|}
\hline Element/Material Type & Property & C.o.V. $(\sigma / \mu)$ & Probability Distribution \\
\hline Isotropic Material & Young's modulus & $8 \%$ & truncated Gaussian \\
& Poisson's ratio & $3 \%$ & \\
& Shear modulus & $12 \%$ & \\
& Mass density & $4 \%$ & \\
\hline Orthotropic Shell & Young's modulus & $8 \%$ & truncated Gaussian \\
Element Material & Poisson's ratio & $3 \%$ & \\
& Shear modulus & $12 \%$ & \\
& Mass density & $4 \%$ & \\
\hline Solid Element An- & Mat. property matrix & $12 \%$ & truncated Gaussian \\
Isotropic Material & Mass density & $4 \%$ & \\
\hline Simple Beam & Section dimension & $5 \%$ & truncated Gaussian \\
& Non-structural mass & $8 \%$ & \\
\hline Layered Composite & Non-structural Mass & $8 \%$ & truncated Gaussian \\
Material & Thickness of plies & $12 \%$ & \\
& Orientation angle & $\sigma=1.5^{\circ}$ & \\
\hline Spring element property & Elastic prop. value & $8 \%$ & truncated Gaussian \\
\hline Shell element & Membrane Thickness & $4 \%$ & truncated Gaussian \\
& Non-structural Mass & $8 \%$ & \\
\hline Spring element & Stiffness & $10 \%$ & truncated Gaussian \\
\hline Concentrated mass & Mass & $3 \%$ & truncated Gaussian \\
\hline Damping & Modal Damping & various & log-normal \\
\hline
\end{tabular}

Particular emphasis is given in this paper to the effect of the uncertainty in the damping on the reliability of the considered structure. Hence, various levels of the uncertainty in the damping have been investigated. In all the cases the damping ratios have been assumed to follow a lognormal distribution and to be mutually independent.

Fig. 2 shows the approximate PDFs of the eigenfrequencies in the frequency range of interest, $[15 \mathrm{~Hz}, 25 \mathrm{~Hz}]$, obtained using direct MCS with 1,500 samples of the full FE model. Clearly, the four eigenfrequencies present in this frequency band are well separated, i.e. there is practically no modal overlapping.

\subsection{Non-parametric probabilistic model}

The construction of the non-parametric model of the INTEGRAL satellite has been performed as outlined in section 2.3, by using the nominal FE model introduced in section 3.2 as the mean model. Based on a convergence analysis performed in [29], the mean model has been reduced using a modal basis of dimension $n=150$. It should be noted that since in the present paper the 


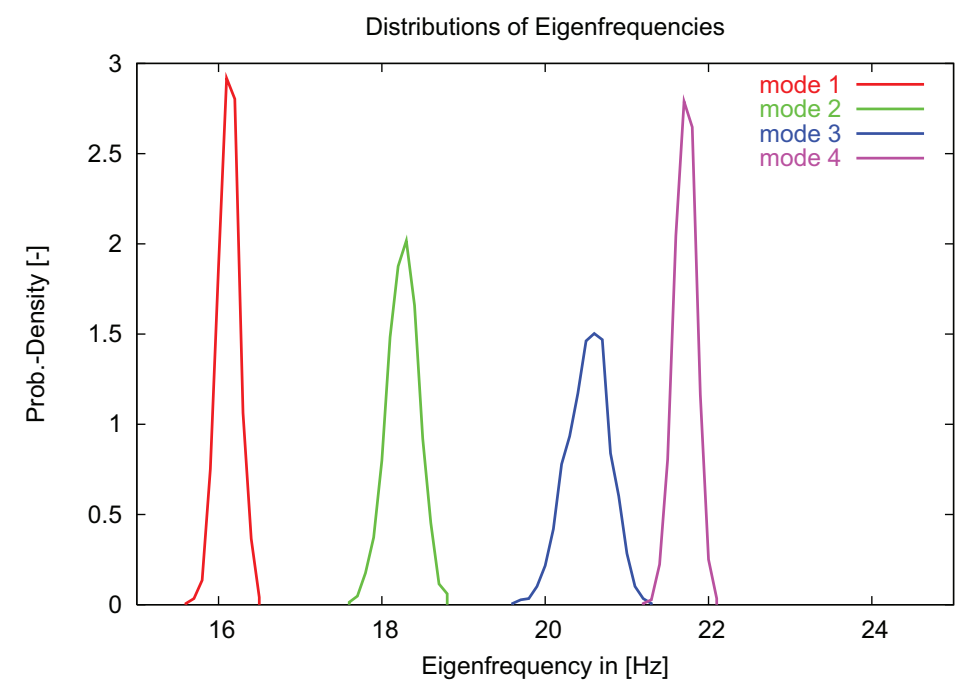

Fig. 2. Approximate PDFs of eigenfrequencies in the frequency range $[15 \mathbf{H z}, 25 \mathbf{H z}]$

analysis is limited to a frequency range of $[5 \mathrm{~Hz}, 25 \mathrm{~Hz}]$, a smaller dimension of the modal basis would have sufficed.

The identification of the dispersion parameters has been performed according to the procedure described in section 2.4. The dispersion parameters of the mass and the stiffness, $\delta_{M}$ and $\delta_{K}$, have been identified by minimizing the difference - i.e. the cost function $J\left(\delta_{M}, \delta_{K}\right)$ introduced in Eq. (19) - between the PDF of the first eigenvalue in the parametric and the non-parametric model, respectively. As shown by the good agreement between the two PDF's in Fig. 3, satisfactory convergence to a minimum of the difference in the PDF's is reached at $\delta_{M}=0.14217$ and $\delta_{K}=0.13487$.

The calibration of the damping dispersion parameter $\delta_{D}$ is discussed in detail in section 3.7.

\subsection{Uncertainty analysis of the FRF}

In the uncertainty analysis of the frequency response function (FRF), the probability distribution of the FRF has been estimated using Monte Carlo simulation. For the parametric model samples of the uncertain parameters $\mathbf{X}$ are generated according to the CDF of $\mathbf{X}$ in Eq. (3), which in the present case study is based on the assumptions stated in section 3.3. For each sample $\mathbf{X}$, the 


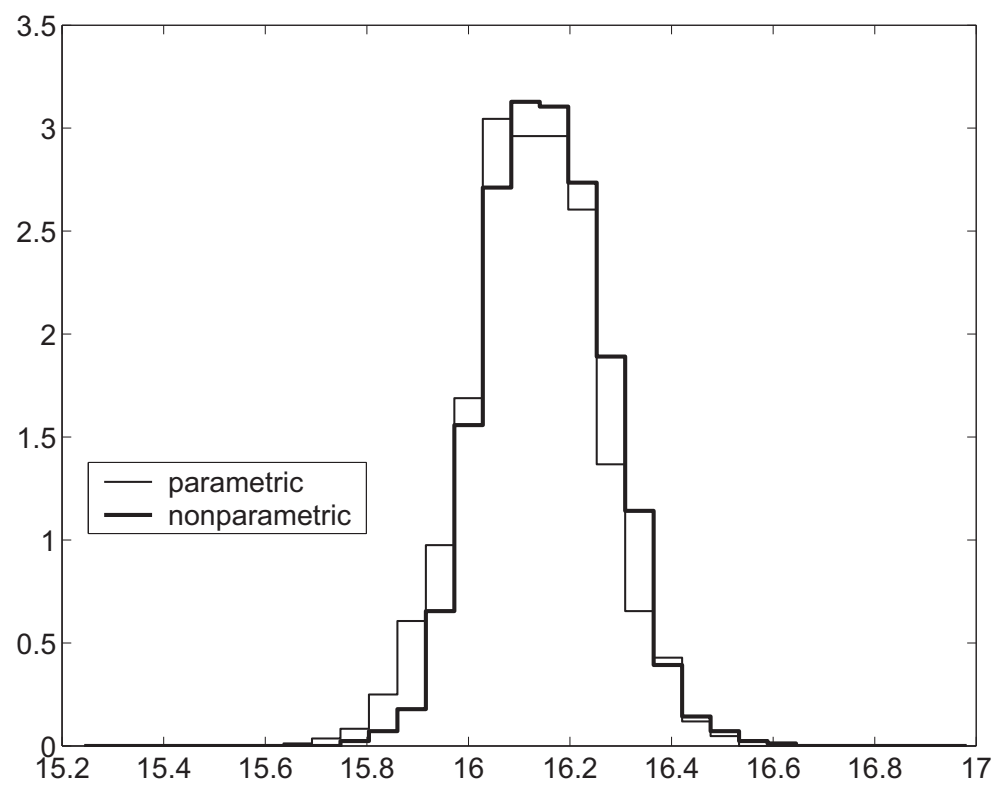

Fig. 3. Identification of dispersion parameters $\delta_{M}$ and $\delta_{K}$ : Graph of the probability distributions $\lambda \mapsto p_{\Lambda_{1}^{\mathrm{par}}}(\lambda)$ for the parametric approach (thin line) and $\lambda \mapsto p_{\Lambda_{1}^{\text {par }}}(\lambda)$ for the non-parametric approach (thick line) with dispersion parameters $\delta_{M}=0.14217$ and $\delta_{K}=0.13487$

structural matrices $\left[\mathbf{M}^{\mathrm{par}}\right],\left[\mathbf{D}^{\mathrm{par}}\right],\left[\mathbf{K}^{\mathrm{par}}\right]$ in Eq. (2) are assembled by the FE solver and Eq. (2) is solved for the FRF $\mathbf{U}^{\text {par }}(\bar{\omega})$.

For the non-parametric model, samples of the matrices $\left[\mathbf{M}_{\mathrm{red}}^{\mathrm{npar}}\right],\left[\mathbf{D}_{\mathrm{red}}^{\mathrm{npar}}\right],\left[\mathbf{K}_{\mathrm{red}}^{\mathrm{npar}}\right]$ in Eq. (14) are generated directly, on the basis of Equations (B.4) and (B.5) and using the identified dispersion parameters. With Equations (14) and (15) the non-parametric FRF $\mathbf{U}^{\text {npar }}(\bar{\omega})$ is then solved for.

From the two ensembles of FRF's, $\left\{\mathbf{U}^{\text {npar }(k)}(\bar{\omega})\right\}_{k=1}^{N_{\text {par }}}$ and $\left\{\mathbf{U}^{\text {par }(k)}(\bar{\omega})\right\}_{k=1}^{N_{\text {npar }}}$, where $N_{\text {par }}$ and $N_{\text {npar }}$ are the number of samples in the parametric and the non-parametric model, respectively, the approximate quantiles of the FRF's can be obtained, as shown in Figures 4 and 5. The shown FRF's refer to the acceleration amplitude at the tip of the SAS booms located at the top of the satellite; the specific node (no. 799921) is marked in Fig. 1.

The two figures refer to different levels of the damping uncertainty. Fig. 4 refers to the case in which the C.o.V. of the modal damping ratios was assumed to be $20 \%$. It is recalled that the damping ratios are mutually independent and log-normally distributed. Using the procedure detailed in section 2.4 (Equations (22) and (23)), this assumption leads to a damping disper- 


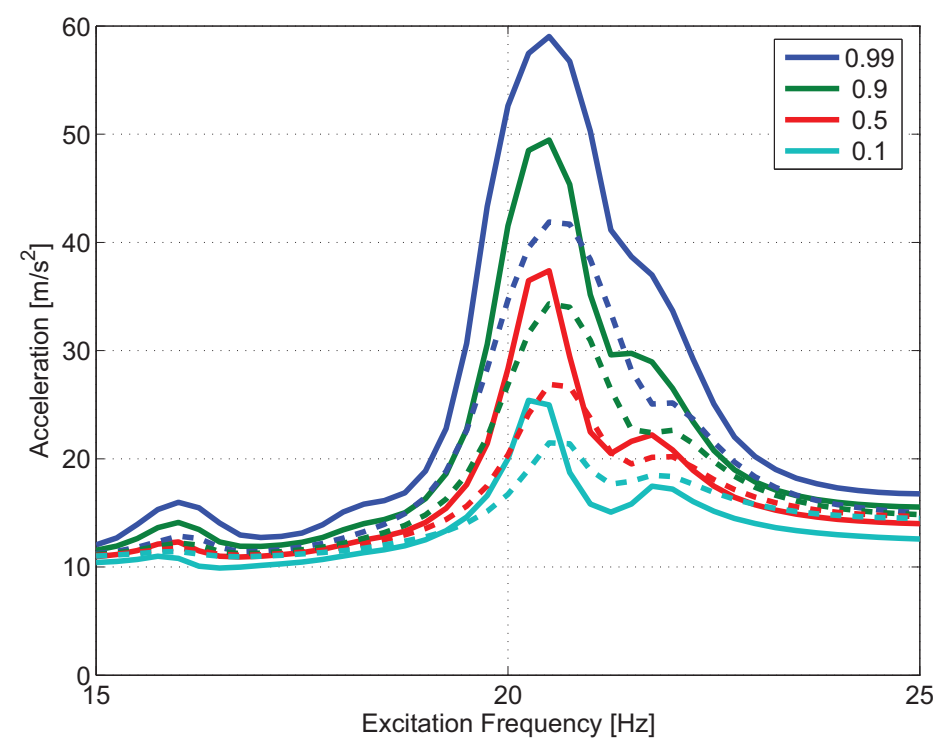

Fig. 4. Acceleration FRF quantiles in the frequency range $[15 \mathrm{~Hz}, 25 \mathrm{~Hz}]$; parametric model (dashed lines, C.o.V of modal damping ratios: $20 \%$ ) and non-parametric model (continuous lines, $\left.\delta_{D}=0.20903\right)$.

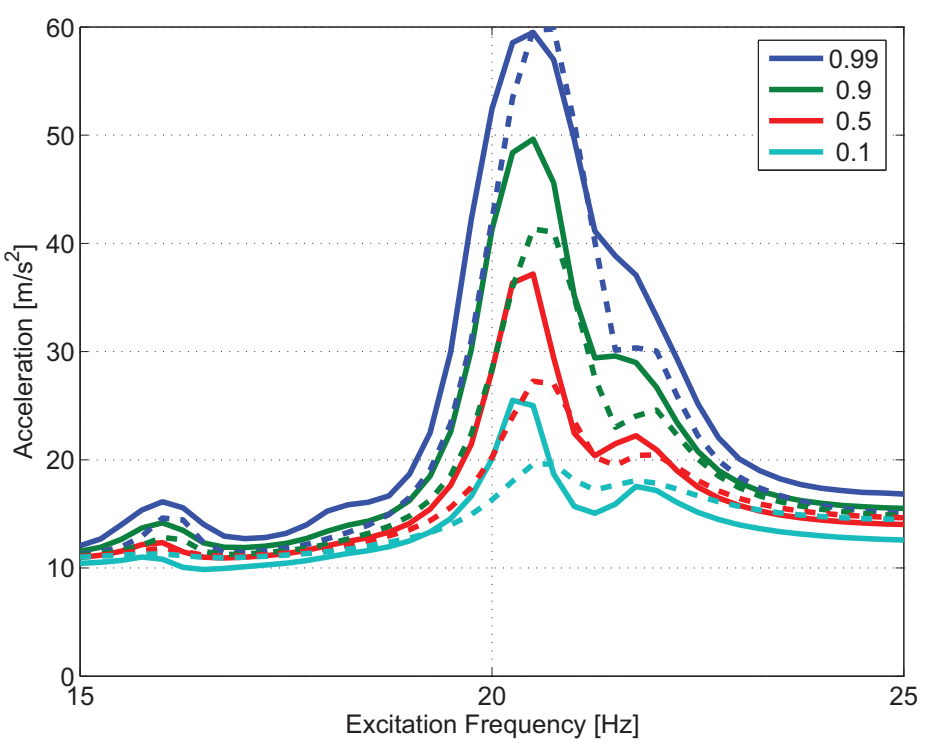

Fig. 5. Acceleration FRF quantiles in the frequency range $[15 \mathrm{~Hz}, 25 \mathrm{~Hz}]$; parametric model (dashed lines, C.o.V of modal damping ratios: $40 \%$ ) and non-parametric model (continuous lines, $\left.\delta_{D}=0.41762\right)$. 
sion parameter of $\delta_{D}=0.20903$. The convergence of the identification procedure for this case (C.o.V. $=20 \%$ ) is shown in one of the sub-figures in Fig. 8.

Fig. 4 shows that in this case the uncertainty in the FRF is significantly greater for the nonparametric model. Indeed the corresponding quantiles (continuous lines) are significantly more spread out than those of the parametric model. This may be interpreted as a consequence of the fact that in the non-parametric model adopted here the model uncertainties are included and this leads to an increase in the FRF uncertainty. The sensitivity of the FRF predictions of the considered satellite structure to model uncertainties in the low frequency range up to $\mathbf{5 0}$ $\mathrm{Hz}$ have already been investigated using the present non-parametric model in an earlier study [29].

Fig. 5 refers to the case in which the C.o.V. of the modal damping ratios $\left\{\Xi_{j}\right\}_{j=1}^{n}$ is assumed to be $40 \%$. In this case the damping dispersion parameter is $\delta_{D}=0.41762$; the convergence of $\delta_{D}$ is shown in Fig. 9. As Fig. 5 shows, the uncertainty in the FRF is now similar for both the parametric and the non-parametric model. In particular, while for the lower probability levels the fractiles of the non-parametric FRF are significantly higher, at least near the peak around 20 $\mathrm{Hz}$, the $99 \%$ fractile of both models is almost identical.

This indicates that in the parametric model the extreme values of the FRF are much more sensitive to the uncertainty in the damping than in the non-parametric model. This phenomenon has been observed not only for the considered satellite structure, but also for other types of structures, such as more simple beam structures modeled with 3-D solid elements.

\subsection{Reliability analysis of the FRF}

While in the previous section the uncertainty of the FRF has been analyzed in general, i.e. in terms of the corresponding probability distribution, the attention is now focussed on the likelihood of extreme FRF levels. This issue is at the core of reliability analysis, in which the probability of exceedance of a given response threshold is of interest. The exceedance of such a threshold is often associated with the failure of a component and its probability therefore termed 


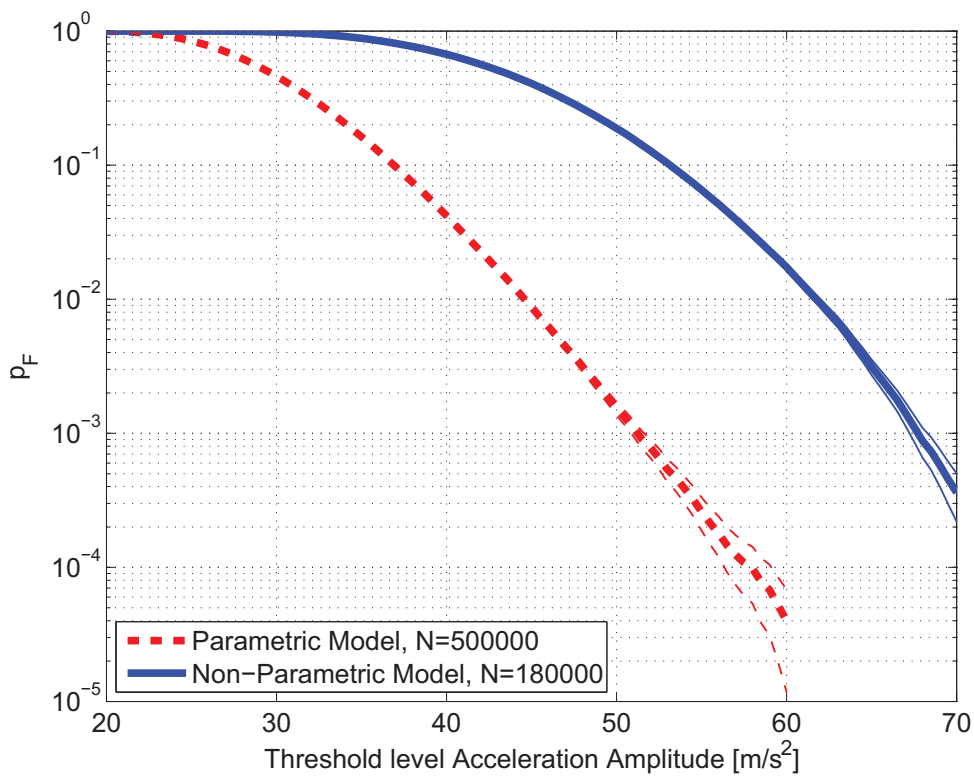

Fig. 6. Probability of acceleration threshold exceedance in the frequency range [5Hz, 25Hz]; parametric model (dashed red line, C.o.V of modal damping ratios: 20\%) and non-parametric model (continuous blue line, $\left.\delta_{D}=0.20903\right)$.

as probability of failure $p_{F}$.

In the present case failure is defined as the exceedance of the acceleration threshold at any excitation frequency in the band $[5 \mathrm{~Hz}, 25 \mathrm{~Hz}]$.

Fig. 6 shows the failure probability as a function of the specified threshold level of the acceleration amplitude. As expected, the failure probability decreases with increasing threshold levels. The figure refers to the case of a C.o.V. of the modal damping ratios of $20 \%$ and shows that for a given threshold level, the failure probability $p_{F}$ is significantly higher for the non-parametric model. It should be noted that the $p_{F}$-estimates have been obtained using direct Monte Carlo simulation (cf. section 2.5.2). The dashed lines indicate the $90 \%$-confidence interval of the $p_{F^{-}}$ estimate.

The situation is clearly different if the uncertainty in the damping increases. Fig. 7 shows the $p_{F}$-estimates for a C.o.V. of $\left\{\Xi_{j}\right\}_{j=1}^{n}$ of $40 \%$. While the $p_{F}$ predicted by the parametric model decreases log-linearly with increasing acceleration threshold, the drop in the $p_{F}$ predicted by the non-parametric model is clearly super-linear. 


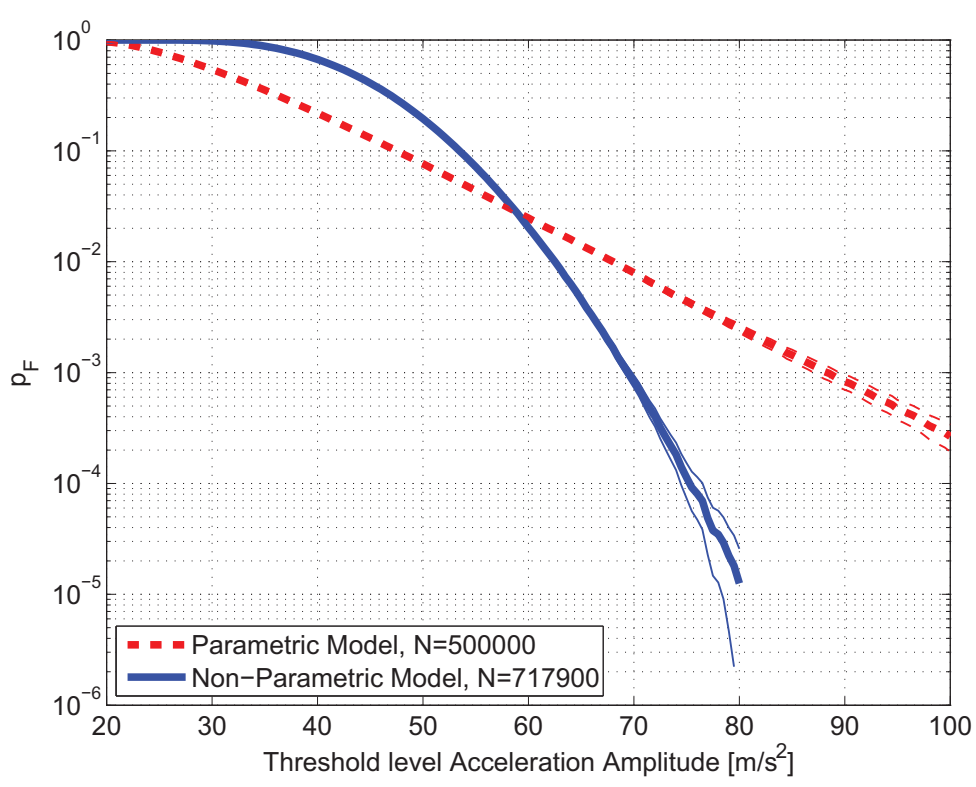

Fig. 7. Probability of acceleration threshold exceedance in the frequency range $[5 \mathrm{~Hz}, 25 \mathrm{~Hz}]$; parametric model (dashed red line, C.o.V of modal damping ratios: 40\%) and non-parametric model (continuous blue line, $\delta_{D}=0.41762$ ).

\subsection{Parameter study - dependence of the reliability on the damping uncertainty}

In view of the strong influence of the level of damping uncertainty on how the failure probability provided by the parametric and the non-parametric model, respectively, relates to each other, a systematic parameter study has been performed, with respect to the C.o.V. of the modal damping ratios in the parametric model. For C.o.V.'s between $5 \%$ and $50 \%$, in steps of $5 \%$, the corresponding damping dispersion parameter has been identified and the non-parametric model constructed. Both for the parametric and the non-parametric model failure probabilities down to approximately $10^{-4}$ have been estimated with Monte Carlo simulation.

Figures 8 and 9 show the convergence after $\mathbf{1 , 5 0 0}$ simulations of the damping dispersion parameter for C.o.V.'s between $5 \%$ and $50 \%$.

Figures 10 and 11 depict the $p_{F}$ estimates of the parametric (red dashed lines) and the nonparametric model (blue continuous lines). Several observations can be made:

- The $p_{F}$-estimates of the non-parametric model are very insensitive to the level of the damping 

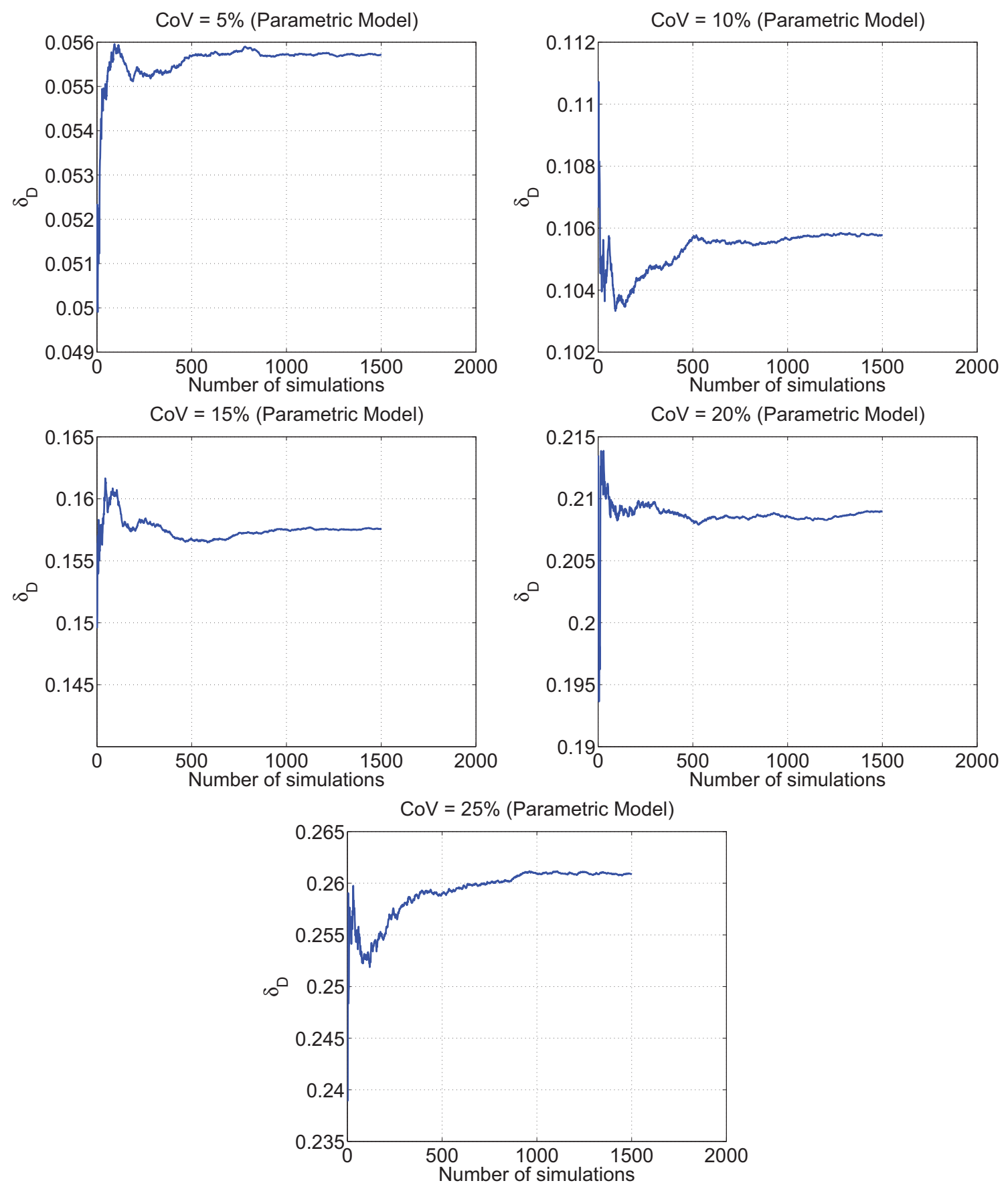

Fig. 8. Convergence of the damping dispersion parameter; C.o.V.'s of the modal damping ratios in the corresponding parametric model: $5 \%-25 \%$

uncertainty. Comparing e.g. the estimates obtained for the case of a C.o.V. of the modal damping ratios of $5 \%$ and for a C.o.V. of $50 \%$, respectively, one concludes that the shift of the $p_{F}$-estimate to the right is hardly perceptible.

- In contrast, the shift to the right of the $p_{F}$-estimates of the parametric model is steady and 

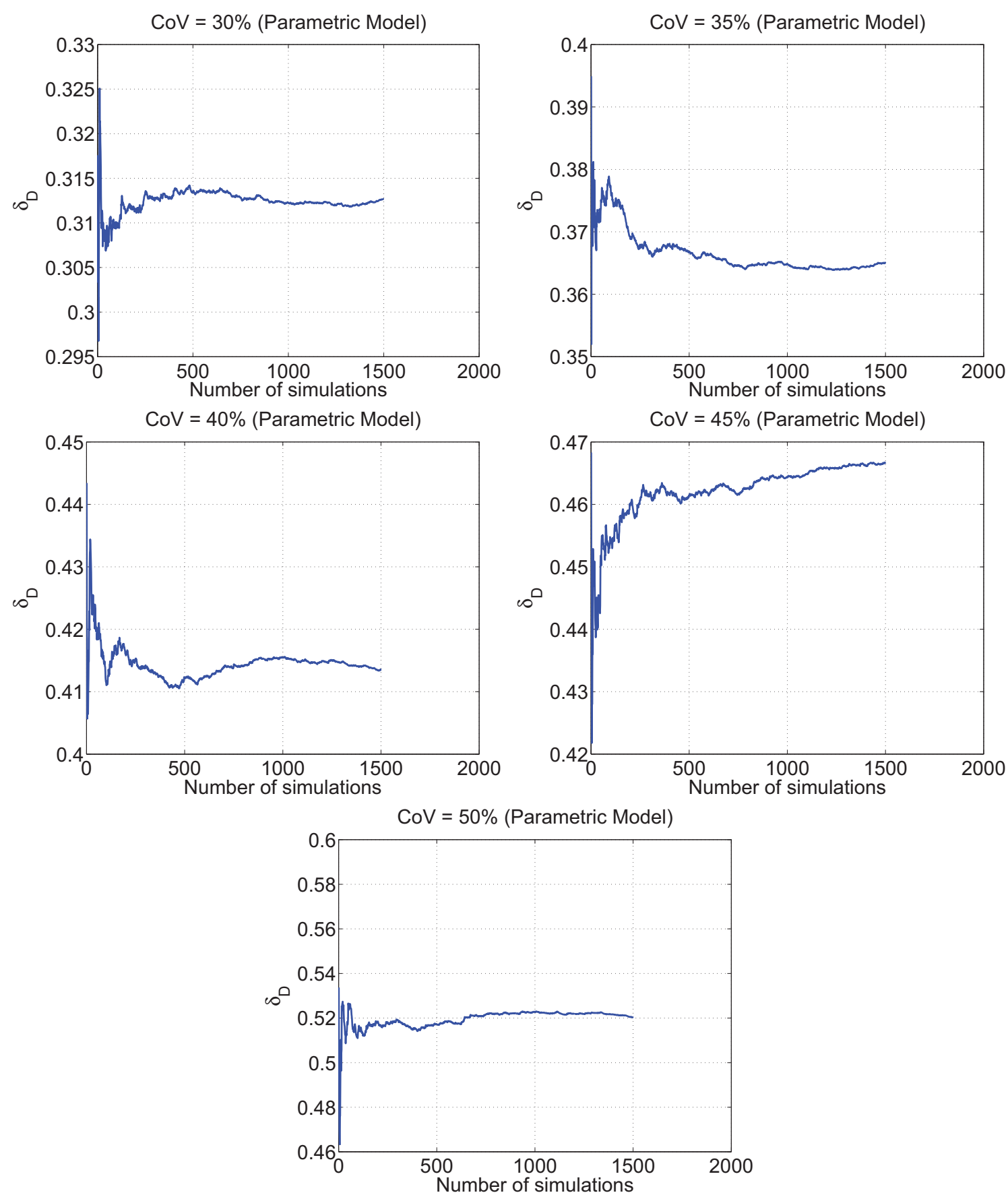

Fig. 9. Convergence of the damping dispersion parameter; C.o.V.'s of the modal damping ratios in the corresponding parametric model: $30 \%-50 \%$

very significant. This indicates that the reliability estimates of the parametric model are very sensitive to the level of uncertainty in the damping.

- For a low uncertainty in the damping (e.g. $5 \%$ ), the $p_{F}$-estimates of the non-parametric model are orders of magnitude more pessimistic (and hence conservative) than those of the paramet- 

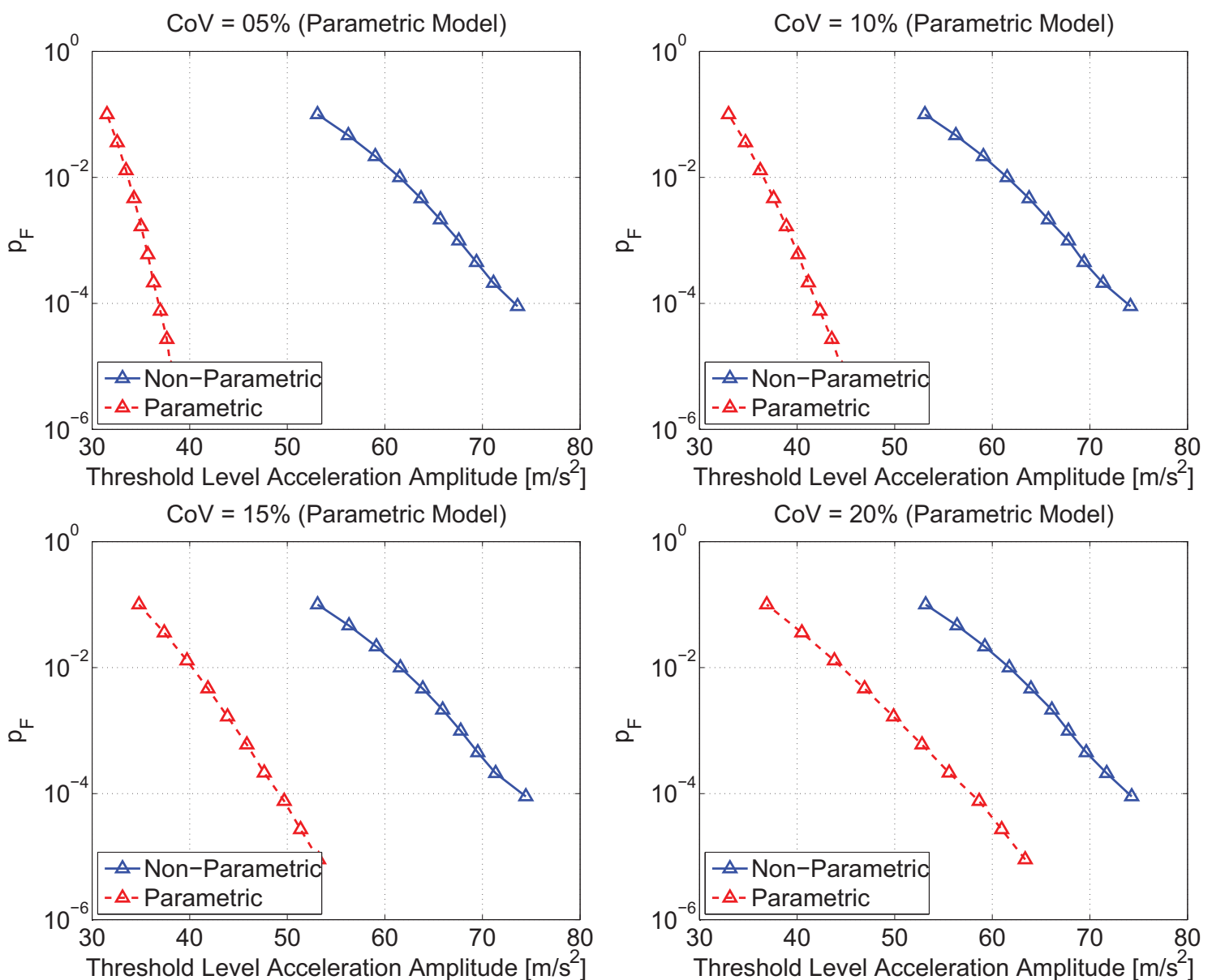

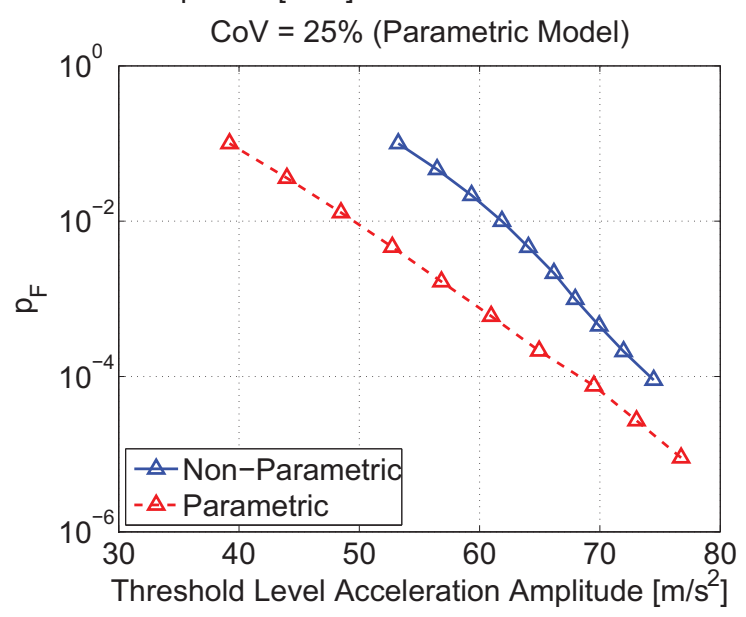

Fig. 10. Probability of acceleration threshold exceedance in the frequency range $[5 \mathrm{~Hz}, 25 \mathrm{~Hz}]$; parametric model (dashed red line, C.o.V of modal damping ratios: 5\%-25\%) and non-parametric model (continuous blue line, $\delta_{D}$ - see corresponding subfigure in Fig. 8).

ric model.

- For a high uncertainty in the damping (e.g. 50\%), the $p_{F}$-estimates of the parametric model 

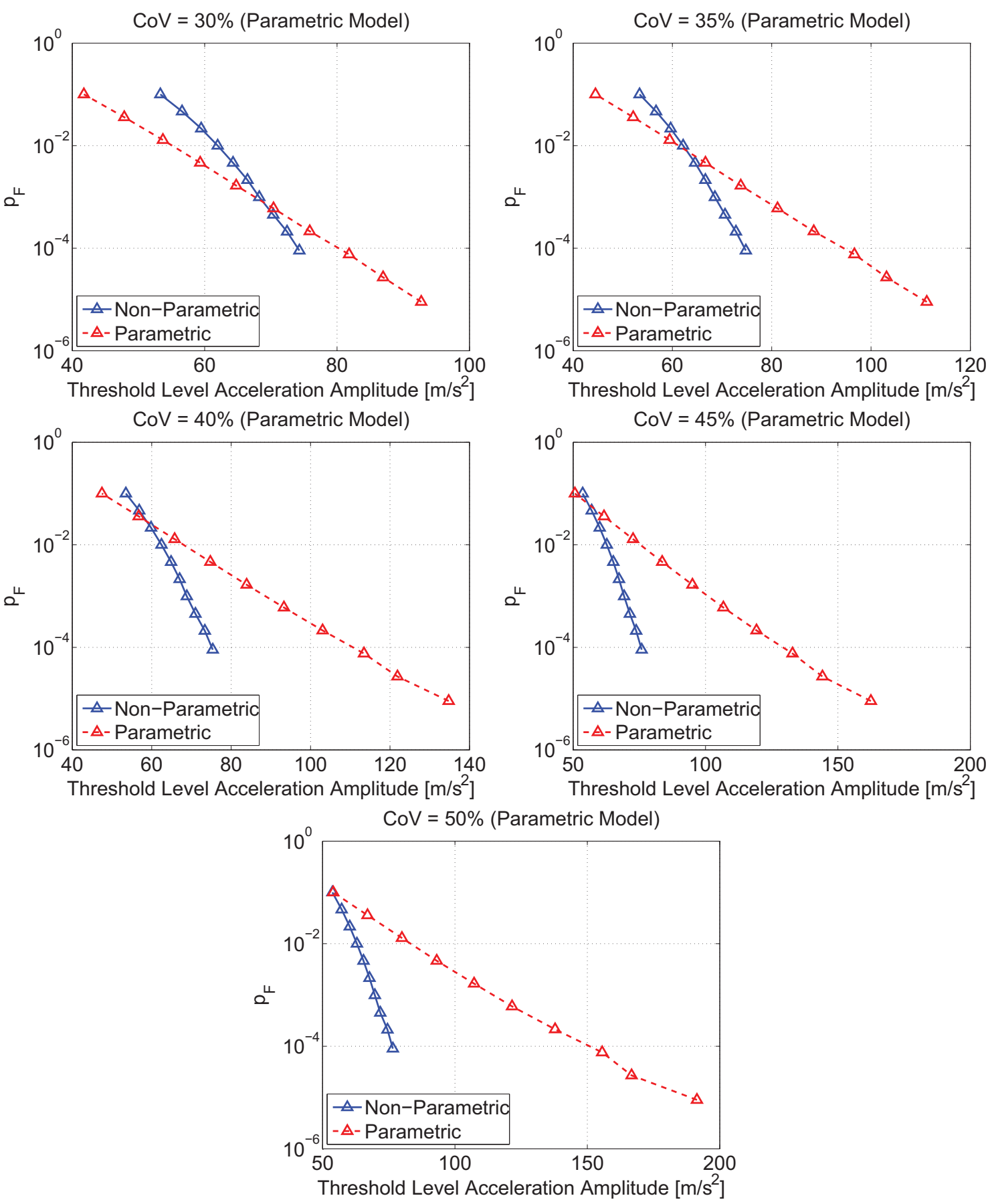

Fig. 11. Probability of acceleration threshold exceedance in the frequency range [5Hz, 25Hz]; parametric model (dashed red line, C.o.V of modal damping ratios: 30\%-50\%) and non-parametric model (continuous blue line, $\delta_{D}$ - see corresponding subfigure in Fig. 9).

are orders of magnitude more pessimistic (and hence conservative) than those of the nonparametric model. 

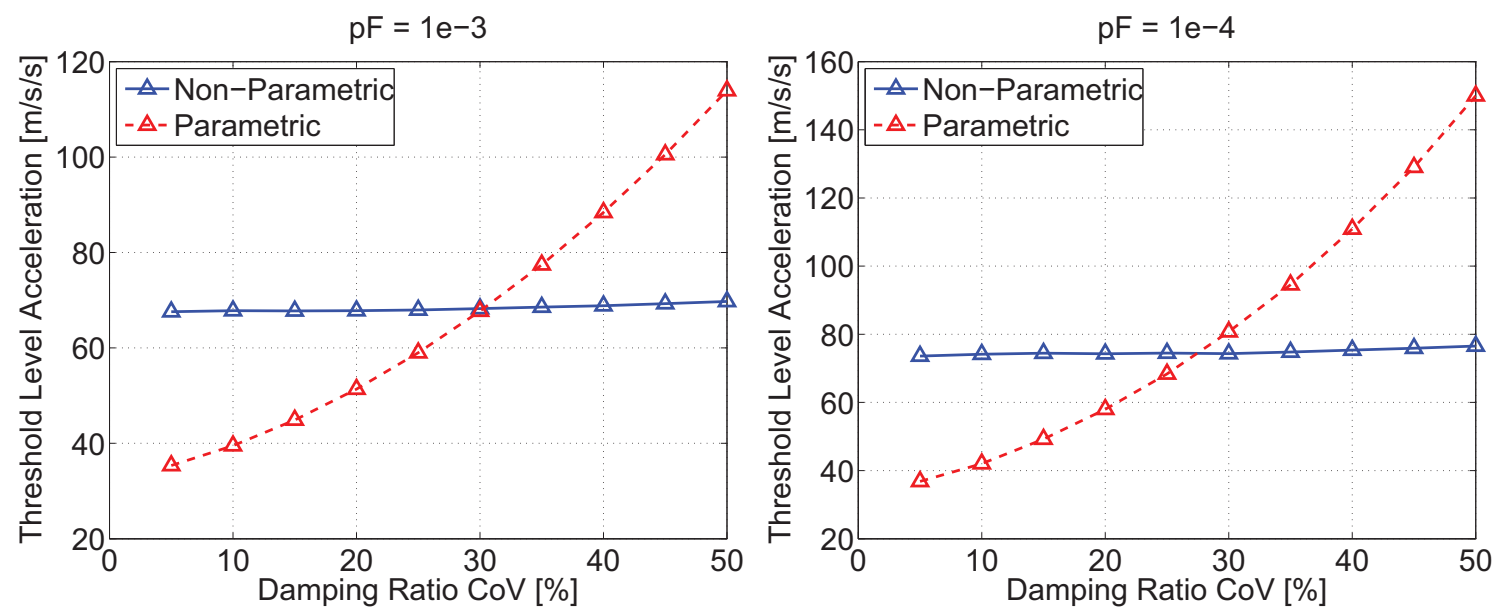

Fig. 12. Acceleration threshold levels leading to $p_{F}=10^{-3}$ (left) and $p_{F}=10^{-4}$ (right) vs. C.o.V of the modal damping ratios of the parametric model; parametric model (red dashed line) and non-parametric probabilistic model (blue continuous line, $\delta_{D}$ - see corresponding sub-figure in Figures 8 and 9)

The above observations are also suggested by Fig. 12, in which the threshold level required to reach a given $p_{F}$ is plotted against the C.o.V. of the damping ratios which underlies the predictions of the parametric and the non-parametric model (in the latter one through the corresponding $\delta_{D}$ ). In particular it is apparent that for the non-parametric model the threshold associated with a given $p_{F}$ is virtually constant and hence entirely insensitive to the level of the damping uncertainty.

Fig. 13 is representative of the accuracy of the failure probability estimates in Figures 10,11 and 12 . It shows the $90 \%$-confidence intervals of the $p_{F}$-estimates for the case in which the nonparametric model has been calibrated based on the assumption of a C.o.V. of $30 \%$ of the modal damping ratios. The number of samples underlying the estimates of the non-parametric model is 100,000 , for all cases investigated in the parameter study. The number of samples used in the estimates of the parametric model is $1,000,000$. Hence the corresponding accuracy is higher than that indicated in Fig. 13. 


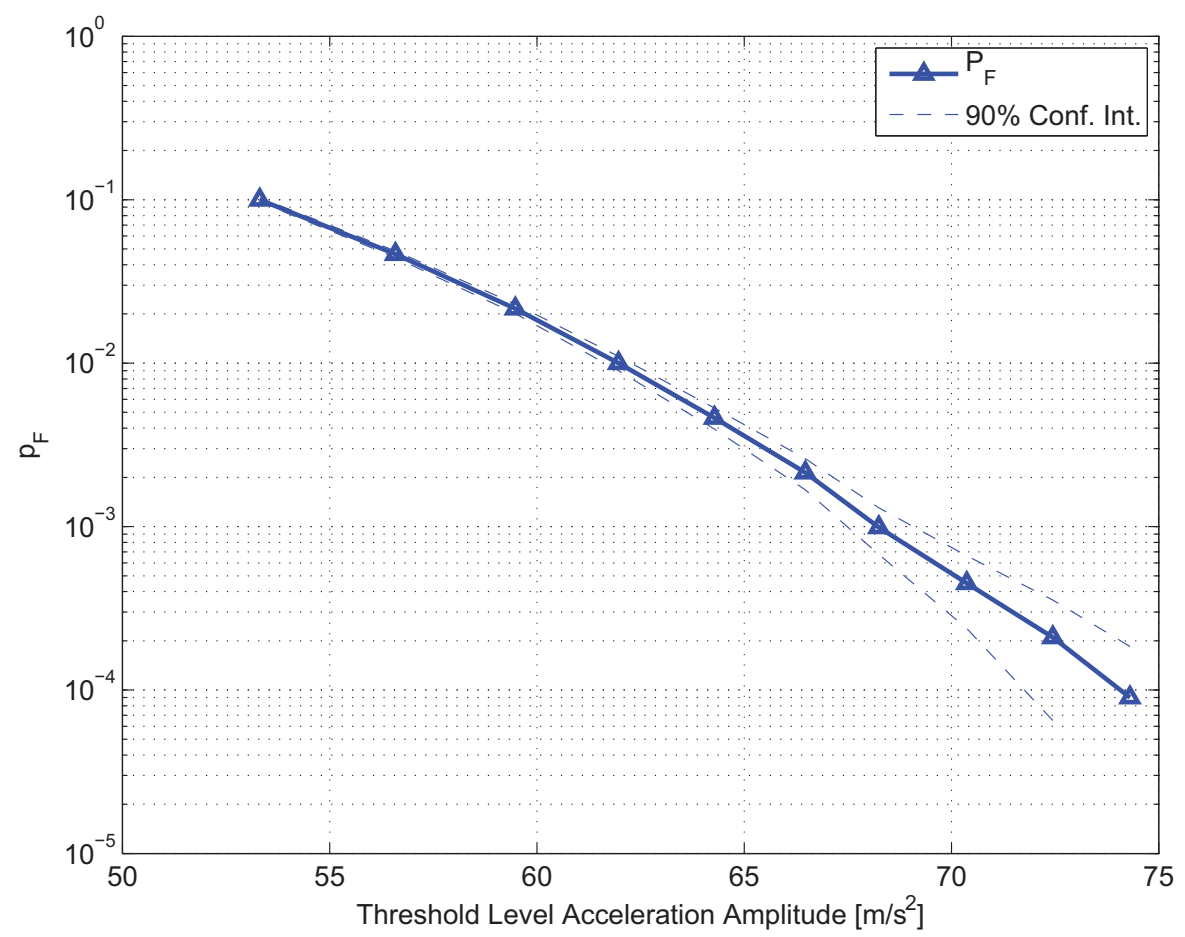

Fig. 13. Probability of acceleration threshold exceedance in the frequency range [ $5 \mathrm{~Hz}, 25 \mathrm{~Hz}]$, with confidence intervals based on Chebyshev's inequality (dashed lines); non-parametric model, $\delta_{D}=0.31277$; 100,000 Monte Carlo samples

\subsection{Computational aspects}

\subsubsection{Meta-model for frequency response analysis}

Given the considerable computational cost for each individual frequency response analysis with the full FE model, as described in section 3.2, a meta-model has been constructed, based on the formulation presented in section A.

The validity of the predictions of the Meta-model is demonstrated in Figures 14 and 15 . The former compares individual samples of the frequency response (in the region of the main peak around $20 \mathrm{~Hz}$ ) obtained with the full model (dashed line) and with the corresponding Metamodel (continuous line). Pairs of lines with the same color were obtained using the same sets of input parameters. The excellent agreement between the respective line pairs shows that for a given set of input parameters, the Meta-model predicts the frequency response with great 


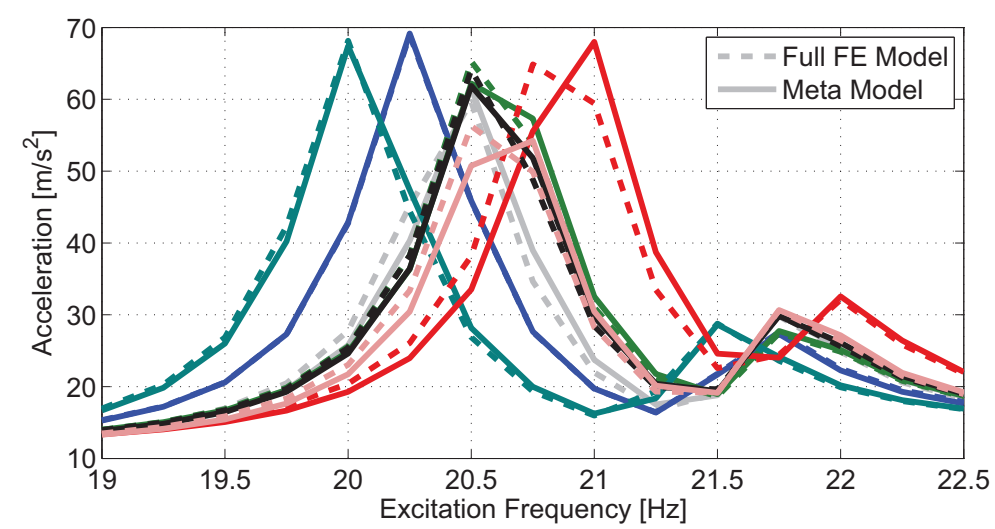

Fig. 14. Verification of meta-model predictions: FRF's for pairwise identical sets of input parameters, full FE model (dashed line) and meta-model (continuous line)

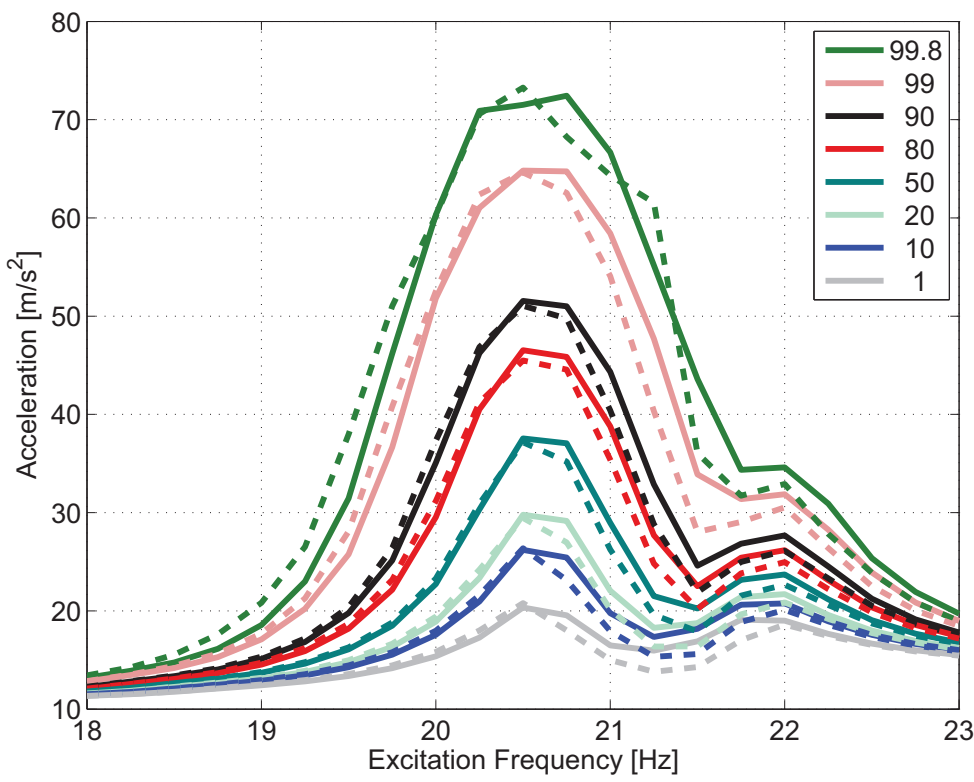

Fig. 15. Verification meta-model predictions: FRF quantiles, full FE model (dashed line) and meta-model (continuous line)

accuracy. It should be noted that the samples of input parameters used to produce the FRF samples shown in Fig. 14 have not been used in the calibration of the meta-model. Hence, in this case, the meta-model is indeed applied as a predictive tool.

Fig. 15 compares the corresponding quantiles, which match very well, too. In view of the excellent agreement between the frequency response of the full FE model and the frequency response predicted by the associated Meta-model, the latter has been considered as verified and was used 
for the reliability estimation of the parametric probabilistic model.

\subsubsection{Parallel reliability analysis}

The parameter study presented in this section involved a conspicuous amount of computations, especially for the non-parametric model. More specifically, the underlying Monte Carlo simulations required a total CPU time in the order of magnitude of 1500 hours (60 CPU-days) on AMD Opteron processors with $2.2 \mathrm{GHz}$.

To reduce this impractical amount of time, the Monte Carlo simulations were performed in parallel on an Opteron cluster. The number of nodes executing the parallel job ranged from 80 to 100 , depending on the availability of cluster nodes. With this arrangement the Monte Carlo simulations of the non-parametric model could be completed in less than one day.

\section{Conclusions}

The present paper investigated the reliability of a satellite structure with respect to the exceedance of a given acceleration threshold of the FRF. This investigation was performed with a parametric probabilistic model, in which the parameters of the nominal FE model are modelled as random variables, and with a non-parametric model, which has been calibrated with respect to the parametric model and which includes the model uncertainties, in addition to the system parameter uncertainties introduced with the parametric model.

The probabilities of exceedance predicted by the two approaches are in general very different; this implies that for the considered problem the inclusion of model uncertainties in the analysis has a great impact on the predicted reliability. As shown in a comprehensive parameter study, the magnitude of this difference depends strongly on the level of uncertainty in the damping. For low levels of uncertainties in the damping, the failure probability estimates are significantly more pessimistic (and hence conservative), if model uncertainties are included in the analysis with a non-parametric model. For high levels of uncertainty in the damping, the opposite has been observed, i.e. in this case the inclusion of model uncertainties, in addition to system 
parameter uncertainties, results in more optimistic failure probability estimates.

The main reason underlying the above observations is that the sensitivity of the predictions to the level of damping uncertainty is highly dependent on whether model uncertainties are considered or not. Indeed, the FRF-predictions are very sensitive to the uncertainty in the modal damping ratios only as long as the uncertainties in the parameters, but not model uncertainties, are considered. If, however, model uncertainties are included by applying a non-parametric model, then the predictions turned out to be very insensitive to the level of the corresponding damping dispersion parameter.

\section{Acknowledgments}

The authors thank the European Space Research and Technology Center (ESTEC) of the European Space Agency (ESA) for providing the satellite FE model. The partial financial support by the AMADEUS European program (Project no. 22/2003 and LAM03) is gratefully acknowledged. The support of the Frankreich-Schwerpunkt at the LFU Innsbruck is also gratefully acknowledged. The usage of the Opteron cluster in the framework of the "Informatik \& Applied Computing" platform (IAC), as well as the support of the IT center (ZID) of the University of Innsbruck are greatly appreciated.

\section{A Meta-model of the parametric probabilistic model}

\section{A.1 Taylor expansion of modal quantities}

The key idea of the proposed meta-model rests on the observation that in the low-frequency domain both the coefficients $C_{j}$ and the eigenfrequencies $\Omega_{j}$ exhibit only a moderate non-linear dependence on the uncertain parameters $\left(X_{1}, \ldots, X_{d}\right)$, provided that there is no modal overlapping, in which case the dependence experiences discontinuities. In the application studied in this manuscript, there is no modal overlapping (cf. Fig. 2) and hence the assumption 
of a moderately non-linear dependence is valid.

Hence it is proposed to approximate the quantities $C_{j}$ and $\Omega_{j}$ in Eq. (11) through a linear approximation. For convenience, the entire set of $C_{j}$ and $\Omega_{j}$ is collected in a vector $\Upsilon$ of generalized modal quantities,

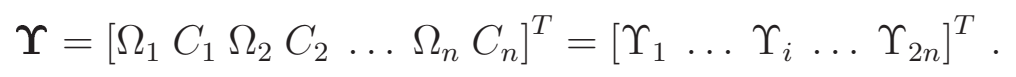

The approximant $\hat{\Upsilon}_{i}$ corresponding to the first-order Taylor expansion of $\Upsilon_{i}$ is then,

$$
\hat{\Upsilon}_{i} \approx \underline{\Upsilon}_{i}+\Upsilon_{i}^{\prime} \cdot(\mathbf{X}-\underline{\mathbf{x}})
$$

where the vector $\Upsilon_{i}^{\prime}$ is the gradient of $\Upsilon_{i}$ with respect to $\mathbf{x}$, evaluated at $\underline{\mathbf{x}}$,

$$
\Upsilon_{i k}^{\prime}=\left.\frac{\partial \Upsilon_{i}}{\partial x_{k}}\right|_{\mathbf{x}=\underline{\mathbf{x}}} .
$$

Once the gradient vector $\Upsilon_{i}^{\prime}$ has been computed, samples of the approximant $\hat{\Upsilon}_{i}$ can be computed extremely fast, by generating samples of the input parameters $\mathbf{X}$ and using Eq. (A.2). Performing this operation for $i \in\{1, \ldots, 2 n\}$ in Eq. (A.1) delivers the approximant $\hat{\Upsilon}$ which corresponds to a sample of approximants $\left\{\hat{\Omega}_{j}\right\}_{j=1}^{n}$ and $\left\{\hat{C}_{j}\right\}_{j=1}^{n}$. Using these approximants in the expansion of the response in Eq. (11) provides an approximation of the sample response, which represents the proposed meta-model,

$$
R_{\mathrm{Meta}}(\bar{\omega})=\sum_{j=1}^{n} \frac{\hat{C}_{j} \bar{\omega}^{p}}{-\bar{\omega}^{2}+i 2 \bar{\omega} \hat{\Omega}_{j} \Xi_{j}+\hat{\Omega}_{j}^{2}} .
$$

\section{A.2 Gradient estimation algorithm}

The evaluation of the gradient formulated in Eq. (A.3) may be viewed as the calibration step in the actual construction of the meta-model. Computationally, this step is also the most expensive part of the meta-model, since in general it is necessary to repeatedly evaluate the full, expensive FE model in order to obtain $\boldsymbol{\Upsilon}_{i}^{\prime}$ for all $i \in\{1, \ldots, 2 n\}$. Indeed, in the context of complex FE models the partial derivative of $\Upsilon_{i}$ is usually computed most expediently by a finite difference 
calculation,

$$
\left.\frac{\partial \Upsilon_{i}}{\partial x_{k}}\right|_{\mathbf{x}=\underline{\mathbf{x}}} \approx \frac{\Upsilon_{i}\left(\underline{\mathbf{x}}+\left[0 \ldots 0 \Delta x_{k} 0 \ldots 0\right]^{T}\right)-\Upsilon_{i}(\underline{\mathbf{x}})}{\Delta x_{k}} .
$$

Alternatively, the partial derivative may be computed analytically, utilizing results on the analytical derivatives of eigenvalues and -vectors $[43,44,45]$. However, the implementation of the analytical derivatives requires significant more implementation effort, as the derivatives have to be extracted from the FE program. In contrast, in the above finite difference formulation one may use the FE program in black-box fashion.

It is clear that the calibration of the meta-model through Eq. (A.3) is particularly expensive if the number $d$ of uncertain parameters $\left(X_{1}, \ldots, X_{d}\right)$ is large, as it is usually the case for FE models of complex structures. In order to speed up this step, a recently introduced statistical gradient estimation method can be relied upon [46,47]. The method is based on Monte Carlo simulation; for any $\Upsilon_{i}$ a corresponding "statistical gradient vector" $\mathrm{s}$ is obtained,

$$
\mathbf{s}=\left[s\left(X_{1}\right) s\left(X_{2}\right) \ldots s\left(X_{d}\right)\right], \quad \text { where } \quad s\left(X_{k}\right)=\left.\sigma_{X_{k}} \frac{\partial \Upsilon_{i}}{\partial x_{k}}\right|_{\mathbf{X}=\underline{\mathbf{x}}} .
$$

Each element $s\left(X_{k}\right)$ of the vector $\mathbf{s}$ may be interpreted as a measure of the relative importance of the corresponding uncertain variable $X_{k}$ for the quantity of interest $\Upsilon_{i}$. As described in [46], an approximation to $\mathrm{s}$ can in most cases be obtained with a rather moderate number of samples $N$, where $N \ll d$. Using this approximation, one can then obtain the partial derivatives of $\Upsilon_{i}$ from Eq. (A.6),

$$
\left.\frac{\partial \Upsilon_{i}}{\partial x_{k}}\right|_{\mathbf{x}=\underline{\mathbf{x}}}=s\left(X_{k}\right) / \sigma_{X_{k}}
$$

The key of the algorithm's efficiency consists in the fact that only those components of $\mathrm{s}$ are evaluated, which appear as the most influential, whereas the remaining ones are neglected.

The essential steps of the algorithm summarized as follows; details may be found in [46,47].

(1) First, $\Upsilon_{i}$ is computed at the mean value of the input parameters, $\Upsilon_{i}=\Upsilon_{i}(\underline{\mathbf{x}})$.

(2) Next, an initial set of $N_{0}$ samples is generated with direct Monte Carlo sampling, using however a reduced standard deviation for the input parameters compared to the true standard deviations $\left\{\sigma_{X_{j}}\right\}_{j=1}^{d}$. This is meant to ensure that $\Upsilon_{i}$ is sampled within the quasilinear 
range with respect to the input parameters.

(3) For each sample $k \in\left\{1, \ldots, N_{0}\right\}$, the modal quantity $\Upsilon_{i}\left(\mathbf{X}^{(k)}\right)$ and the corresponding deviation from $\underline{\Upsilon}_{i}$ are evaluated, i.e. $b^{(k)}=\Upsilon_{i}\left(\mathbf{X}^{(k)}\right)-\Upsilon_{i}$.

(4) With the available set of samples, the sample correlation between each input parameter and $\Upsilon_{i}$ is evaluated. A ranking of the relative importance of all uncertain input parameters $\left\{X_{j}\right\}_{j=1}^{d}$ is then established, based on the absolute value of the correlation.

(5) For the $L$ most important variables the corresponding partial derivative is determined with by finite differences, i.e. using Eq. (A.5). The integer value $L$ is user-defined; for the sake of efficiency $L \ll d$, as a rule of thumb $L=10$ is suggested.

(6) The actual relative importance is assessed with Eq. (A.6) and compared with the "average importance". If it is below that the so-called failure counter variable is incremented; indeed the fact that a parameter which was estimated to be of major importance turns out to be in fact below the "average importance" is considered a failure. Whenever the failure counter exceeds a certain user-defined threshold value (e.g. 10), an additional set of MCS-samples is generated and the algorithm proceeds from there.

(7) The influence of those parameters, whose partial derivatives have been computed based on the importance ranking, is removed from the ensemble of deviations, $\left\{b^{(k)}\right\}_{k=1}^{N_{0}}$. The ensemble of the remaining deviations is denoted as $\left\{\tilde{b}^{(k)}\right\}_{k=1}^{N_{0}}$.

(8) Next, the exit criterion is probed: (1) if the achieved accuracy exceeds the user-defined accuracy threshold, i.e. if $\epsilon=\sqrt{1-\frac{\|\tilde{\mathbf{b}}\|}{\|b\|}} \geq \epsilon_{\min }$, convergence has been reached; a typical value for $\epsilon_{\min }$ is 0.95 ; it should be noted that convergence is guaranteed after evaluating at most $d$ (i.e. all ) partial derivatives; (2) if the accuracy of the gradient estimate is not sufficient, the relative importance of additional parameters has to be evaluated.

\section{A.3 Retrieval of modal quantities from FE code output}

Unless the analyst has access to the source code of the FE code used for the dynamic analysis, the following practical problem arises in the calibration of the meta-model: for a given response quantity of interest $R_{\text {Full }}(\bar{\omega})$, the modal quantities $\left\{C_{j}\right\}_{j=1}^{n}$ in Eq. (11) cannot be obtained directly from the output of the FE code. 
However, it is possible to compute the set $\left\{C_{j}\right\}_{j=1}^{n}$ for a given sample, by post-processing the available set of responses,

$$
\left\{R_{\mathrm{Full}}\left(\bar{\omega}_{m}\right)\right\}_{m=1}^{M}, \quad \bar{\omega}_{m}=\bar{\omega}_{1}+(m-1) \Delta \bar{\omega} .
$$

where $\bar{\omega}_{1}$ and $\bar{\omega}_{M}$ are the lower and the upper bound, respectively, of the considered frequency band $\mathbb{B}, \Delta \bar{\omega}$ is the frequency increment and $M$ is the total number of frequencies at which the FRF is computed. With reference to Eq. (11), the following set of equations can be formulated for a given sample,

$$
R_{\mathrm{Full}}\left(\bar{\omega}_{m}\right)=\sum_{j=1}^{n} A_{j}\left(\bar{\omega}_{m}\right) C_{j} \quad \forall m \in\{1, \ldots, M\}, \quad \text { where } \quad A_{j}=\frac{\bar{\omega}^{p}}{-\bar{\omega}^{2}+i 2 \bar{\omega} \Omega_{j} \Xi_{j}+\Omega_{j}^{2}} .
$$

It should be noted that for each sample the set $\left\{A_{j}\right\}_{j=1}^{n}$ is known, provided the FE code outputs the eigenfrequencies $\left\{\Omega_{j}\right\}_{j=1}^{n}$; the (random) modal damping ratios $\left\{\Xi_{j}\right\}_{j=1}^{n}$ are user defined and thus also known. The above set of equations may be written in matrix form,

$$
[\mathbf{A}] \mathbf{C}=\mathbf{R}, \quad \text { where } \quad[\mathbf{A}] \in \mathbb{C}^{M, n}, \quad \mathbf{C} \in \mathbb{C}^{n, 1}, \quad \mathbf{R} \in \mathbb{C}^{M, 1}
$$

and where,

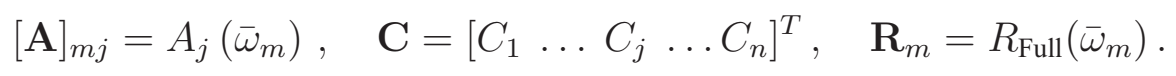

The above overdetermined system in C may be solved using singular value decomposition. It should be noted that the accuracy of the above introduced response representation in terms of $\mathrm{C}$ is satisfactory only within the frequency range considered in this manuscript. In particular, its accuracy appears to be very sensitive to modal overlapping.

\section{B Probability distribution of the random matrices}

In the following, $[\mathbf{G}]$ denotes any one of the matrices $\left[\mathbf{G}_{M}\right],\left[\mathbf{G}_{D}\right]$ and $\left[\mathbf{G}_{K}\right]$ and $\delta$ denotes the corresponding dispersion parameter. The probability density function of random matrix $[\mathbf{G}]$ with 
respect to the volume element

$$
\tilde{d} G=2^{n(n-1) / 4} \prod_{1 \leq i \leq j \leq n} d G_{i j}
$$

is given by,

$$
p_{[\mathbf{G}]}([G])=\mathbb{1}_{\mathbb{M}_{n}^{+}(\mathbb{R})}([G]) \times C_{G} \times(\operatorname{det}([G]))^{\left(1-\delta^{2}\right)\left(2 \delta^{2}\right)^{-1}(n+1)} \times e^{-(n+1)\left(2 \delta^{2}\right)^{-1} \operatorname{tr}[G]},
$$

in which $\mathbb{1}_{\mathbb{M}_{n}^{+}(\mathbb{R})}$ denotes the indicatrix function indexed on the set of all the symmetric $(n \times$ $n$ ) real positive-definite matrices, det and $t r$ are the determinant and the trace of the matrix, respectively. Furthermore $C_{G}$ is the positive constant,

$$
C_{G}=\frac{(2 \pi)^{-n(n-1) / 4}\left(\frac{n+1}{2 \delta^{2}}\right)^{n(n+1)\left(2 \delta^{2}\right)^{-1}}}{\prod_{j=1}^{n} \Gamma\left(\frac{n+1}{2 \delta^{2}}+\frac{1-j}{2}\right)},
$$

in which $\Gamma(z)$ is the gamma function defined for all $z>0$ by $\Gamma(z)=\int_{0}^{\infty} t^{z-1} e^{-t} d t$. Eq. $(B .2)$ shows that the entries $[\mathbf{G}]_{j k}$ of random matrix $[\mathbf{G}]$ are dependent random variables.

The following algebraic representation of random positive-definite symmetric real matrix $[\mathbf{G}]$ allows a procedure for the Monte Carlo numerical simulation of random matrix $[\mathbf{G}]$ to be defined. The random matrix $[\mathbf{G}]$ is written as,

$$
[\mathbf{G}]=\left[\mathbf{L}_{G}\right]^{T}\left[\mathbf{L}_{G}\right],
$$

in which $\left[\mathbf{L}_{G}\right]$ is an $(n \times n)$ upper triangular random matrix resulting from the Cholesky factorisation such that

(1) random variables $\left\{\left[\mathbf{L}_{G}\right]_{j j^{\prime}}, j \leq j^{\prime}\right\}$ are independent;

(2) for $j<j^{\prime}$, real-valued random variable $\left[\mathbf{L}_{G}\right]_{j j^{\prime}}$ can be written as $\left[\mathbf{L}_{G}\right]_{j j^{\prime}}=\sigma_{n} U_{j j^{\prime}}$ in which $\sigma_{n}=\delta(n+1)^{-1 / 2}$ and where $U_{j j^{\prime}}$ is a real-valued Gaussian random variable with zero mean and variance equal to 1 ;

(3) for $j=j^{\prime}$, positive-valued random variable $\left[\mathbf{L}_{G}\right]_{j j}$ can be written as $\left[\mathbf{L}_{G}\right]_{j j}=\sigma_{n} \sqrt{2 V_{j}}$ in which $\sigma_{n}$ is defined above and where $V_{j}$ is a positive-valued gamma random variable whose 
probability density function $p_{V_{j}}(v)$ with respect to $d v$ is written as

$$
p_{V_{j}}(v)=\mathbb{1}_{\mathbb{R}^{+}}(v) \frac{1}{\Gamma\left(\alpha_{n, j}\right)} v^{\alpha_{n, j}-1} e^{-v} \quad, \quad \alpha_{n, j}=\frac{n+1}{2 \delta^{2}}+\frac{1-j}{2} .
$$




\section{References}

[1] C. Soize, A nonparametric model of random uncertainties for reduced matrix models in structural dynamics, Probabilistic Engineering Mechanics 15 (2000) 277-294.

[2] C. Soize, Maximum entropy approach for modeling random uncertainties in transient elastodynamics, Journal Of The Acoustical Society Of America 109 (2001) 1979-1996.

[3] C. Soize, A comprehensive overview of a non-parametric probabilistic approach of model uncertainties for predictive models in structural dynamics, Journal of Sound and Vibration 288 (3) (2005) 623-652.

[4] C. Soize, Random matrix theory for modeling uncertainties in computational mechanics, Computer Methods In Applied Mechanics And Engineering 194 (2005) 1333-1366.

[5] E. Henley, H. Kumamoto, Reliability Engineering and Risk Assessment, Prentice-Hall, Inc., Englewood Cliffs, New Jersey, 1981.

[6] T. Bedford, R. Cooke, Probabilistic Risk Analysis - Foundations and Methods, Cambridge University Press, Cambridge, UK, 2001.

[7] G. Schuëller, Einführung in die Sicherheit und Zuverlässigkeit von Tragwerken, Wilhelm Ernst und Sohn, Berlin, München, 1981.

[8] U. Hauptmanns, W. Werner, Engineering risks - evaluation and valuation, Springer-Verlag, Berlin, 1991.

[9] M. Shinozuka, C. Astill, Random eigenvalue problems in structural analysis, AIAA Journal 10 (4) (1972) 456-462.

[10] R. Ibrahim, Structural dynamics with parameter uncertainties, Applied Mechanical Reviews 40 (3) (1987) 309-328.

[11] R. Ghanem, P. Spanos, Stochastic Finite Elements: A Spectral Approach, Springer Verlag, Berlin, 1991.

[12] W. Iwan, H. Jensen, On the dynamic response of continuous systems including model uncertainty, Journal of Applied Mechanics, Transactions ASME 60 (2) (1993) 484-490. 
[13] R. Singh, C. Lee, Frequency response of linear systems with parameter uncertainties, Journal of Sound and Vibration 168 (1) (1993) 507-516.

[14] R. Ghanem, Ingredients for a general purpose stochastic finite elements implementation, Computer Methods in Applied Mechanics and Engineering 168 (1-4) (1999) 19-34.

[15] G. I. Schuëller, Computational stochastic mechanics - recent advances, Computers \& Structures $79(22-25)(2001) 2225-2234$.

[16] H. J. Pradlwarter, G. I. Schuëller, G. Székely, Random eigenvalue problems for large systems, Computers \& Structures 80 (2002) 2415-2424.

[17] C. A. Schenk, G. I. Schuëller, Uncertainty Assessment of Large Finite Element Systems, SpringerVerlag, Berlin/Heidelberg/New York, 2005.

[18] C. Papadimitriou, J. Beck, L. Katafygiotis, Asymptotic expansions for reliability and moments of uncertain systems, Journal of Engineering Mechanics-ASCE 123 (12) (1997) 1219-1229.

[19] S.-K. Au, J. Beck, Estimation of small failure probabilities in high dimensions by subset simulation, Probabilistic Engineering Mechanics 16 (4) (2001) 263-277.

[20] G. I. Schuëller, H. J. Pradlwarter, P. Koutsourelakis, A critical appraisal of reliability estimation procedures for high dimensions, Probabilistic Engineering Mechanics 19 (4) (2004) 463-474.

[21] H. J. Pradlwarter, M. F. Pellissetti, C. A. Schenk, G. I. Schuëller, A. Kreis, S. Fransen, A. Calvi, M. Klein, Realistic and efficient reliability estimation for aerospace structures, Computer Methods in Applied Mechanics and Engineering 194 (2005) 1597-1617.

[22] G. I. Schuëller, H. J. Pradlwarter, Benchmark study on reliability estimation in higher dimensions of structural systems - an overview, Structural Safety 29 (3) (2007) 167-182.

[23] J. Beck, L. Katafygiotis, Updating models and their uncertainties. i: Bayesian statistical framework, Journal of Engineering Mechanics 124 (4) (1998) 455-461.

[24] C. Papadimitriou, J. Beck, L. Katafygiotis, Updating robust reliability using structural test data, Probabilistic Engineering Mechanics 16 (2001) 103-113.

[25] T. Igusa, S. Buonopane, B. Ellingwood, Bayesian analysis of uncertainty for structural engineering applications, Structural Safety 24 (2002) 165-186. 
[26] O. Ditlevsen, Model uncertainty in structural reliability, Structural Safety 1 (1982) 73-86.

[27] A. Der Kiureghian, Measures of structural safety under imperfect states of knowledge, Journal of Structural Engineering - ASCE 115 (5) (1989) 1119-1140.

[28] R. Menezes, G. I. Schuëller, On structural reliability assessment considering mechanical model uncertainties, in: H. Natke, Y. Ben-Haim (Eds.), Uncertainty: Models and Measures, Vol. 99 of Mathematical Research Series, Akademie Verlag, 1997, pp. 173-186.

[29] E. Capiez-Lernout, M. Pellissetti, H. Pradlwarter, G. I. Schuëller, C. Soize, Data and model uncertainties in complex aerospace engineering systems, Journal of Sound and Vibration 295 (35) (2006) 923-938.

[30] C. Shannon, A mathematical theory of communication, Bell System Technology Journal (1948) $379-423$ and 623-659.

[31] M. Friswell, J. Mottershead, Finite Element Model Updating in Structural Dynamics, Kluwer Academic Publisher, 1995.

[32] E. Capiez-Lernout, C. Soize, Nonparametric modeling of random uncertainties for dynamic response of mistuned bladed-disks., ASME Journal of Engineering for Gas Turbines and Power 126 (3) (2004) 610-618.

[33] R. Rubinstein, Simulation and the Monte Carlo Method, John Wiley \& Sons, New York, Chichester, Brisbane, Toronto, 1981.

[34] C. Winkler, T. J.-L. Courvoisier, G. D. Cocco, N. Gehrels, A. Giménez, S. Grebenev, W. Hermsen, J. M. Mas-Hesse, F. Lebrun, N. Lund, G. G. C. Palumbo, J. Paul, J.-P. Roques, H. Schnopper, V. Schönfelder, R. Sunyaev, B. Teegarden, P. Ubertini, G. Vedrenne, A. J. Dean, The integral mission, Astronomy \& Astrophysics 411 (1) (2003) L1-L6, special Letters issue on: First science with INTEGRAL.

[35] M. Oxfort, Integral - plm payload module structure fem description, Tech. Rep. INT-RP-OCW-0002, 2nd edition, Oerlikon-Contraves BU Space, Zürich-Seebach, Switzerland (December 1997).

[36] C. Moreno, Integral - service module structure mathematical model description, Tech. Rep. INTTN-CAS-1002, 1st edition, CASA Space Division, Madrid, Spain (April 1998). 
[37] M. Notarnicola, A. Paron, L. Tizzani, E. Evans, Integral - structural mathematical model description and dynamic analysis results, Tech. Rep. INT-TN-Al-0089, Issue 2, Alenia Aerospazio Space Division, Turin, Italy (April 15 1998).

[38] MSC.NASTRAN, Version 70.7, MSC.Software Corporation (2001).

[39] P. Esnault, M. Klein, Factors of safety and reliability - present guidelines \& future aspects, in: Proceedings of the Conference on Spacecraft Structures, Materials \& Mechanical Testing, SP-386, European Space Agency, Nordwijk, The Netherlands, 1996, pp. 109-119.

[40] M. Klein, G. Schuëller, P. Deymarie, M. Macke, P. Courrian, R. S. Capitanio, Probabilistic approach to structural factors of safety in aerospace, in: Proceedings of the International Conference on Spacecraft Structures and Mechanical Testing, Cépadués-Editions, Paris, France, 1994, pp. 679 693.

[41] G. Székely, W. Teichert, C. Brenner, H. Pradlwarter, M. Klein, G. Schuëller, Practical procedures for reliability estimation of spacecraft structures and their components, J. AIAA 36 (8) (1998) 1509 $-1515$.

[42] S. Simonian, Survey of spacecraft damping measurements: Applications to electro-optic jitter problems, in: The Role of Damping in Vibration and Noise Control, Vol. DE-Vol. 5, ASME Publication, 1987, pp. 287-292.

[43] R. B. Nelson, Simplified calculation of eigenvector derivatives, AIAA Journal 14 (9) (1976) 12011205.

[44] I. Ojalvo, Efficient computation of mode-shape derivatives for large dynamics systems, AIAA Journal 25 (10) (1987) 1386-1390.

[45] W. C. Mills-Curran, Calculation of eigenvector derivatives for structures with repeated eigenvalues, AIAA Journal 26 (7) (1988) 867-871.

[46] H. J. Pradlwarter, Relative importance of uncertain structural parameters , part I: Algorithm, Computational Mechanics 40 (4) (2007) 627-635.

[47] M. F. Pellissetti, H. J. Pradlwarter, G. I. Schuëller, Relative importance of uncertain structural parameters , part II: Applications, Computational Mechanics 40 (4) (2007) 637-649. 